\title{
Cervical Epidural Electrical Stimulation Restores Voluntary Arm Control In Paralyzed Monkeys
}

\section{Marco Capogrosso (D MCAPO@pitt.edu )}

University of Pittsburgh

\section{Beatrice Barra}

University of Fribourg

\section{Sara Conti}

University of Fribourg

\section{Matthew Perich}

Icahn School of Medicine at Mount Sinai https://orcid.org/0000-0001-9800-2386

Katie Zhuang

University of Fribourg

Giuseppe Schiavone

École Polytechnique Fédérale de Lausanne https://orcid.org/0000-0001-7121-9825

\section{Florian Fallegger}

Ecole Polytechnique Federale de Lausanne

\section{Katia Galan}

Ecole Polytechnique Federale de Lausanne

\section{Nicholas James}

Ecole Polytechnique Federale de Lausanne

\section{Quentin Barraud}

SWISS FEDERAL INSTITUTE OF TECHNOLOGY (EPFL) https://orcid.org/0000-0003-0894-1959

\section{Maude Delacombaz}

University of Fribourg

\section{Melanie Kaeser}

University of Fribourg

\section{Eric Rouiller}

Unit of Physiology

\section{Tomislav Milekovic}

University of Geneva https://orcid.org/0000-0001-6769-6506

\section{Stephanie Lacour}

Ecole Polytechnique Fédérale de Lausanne (EPFL) https://orcid.org/0000-0001-9075-4022 Jocelyne Bloch

Centre Hospitalier Universitaire Vaudois (CHUV)

\section{Gregoire Courtine}


École Polytechnique Fédérale de Lausanne https://orcid.org/0000-0002-5744-4142

\section{Article}

Keywords: paralysis, arm motor control

Posted Date: December 28th, 2020

DOI: https://doi.org/10.21203/rs.3.rs-123709/v1

License: (c) (1) This work is licensed under a Creative Commons Attribution 4.0 International License. Read Full License 


\section{CERVICAL EPIDURAL ELECTRICAL STIMULATION RESTORES VOLUNTARY ARM 2 CONTROL IN PARALYZED MONKEYS}

3 B. Barra ${ }^{1,2, *}$, S. Conti ${ }^{1, *}$, M.G. Perich ${ }^{3}$, K. Zhuang ${ }^{1}$, G. Schiavone ${ }^{4}$, F. Fallegger ${ }^{4}$, K. Galan ${ }^{5}$, N. D.

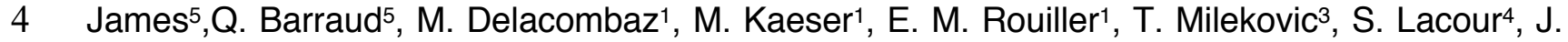
5 Bloch ${ }^{6,7}$, G. Courtine ${ }^{5,6,7}$ and M. Capogrosso ${ }^{1,2,8}$

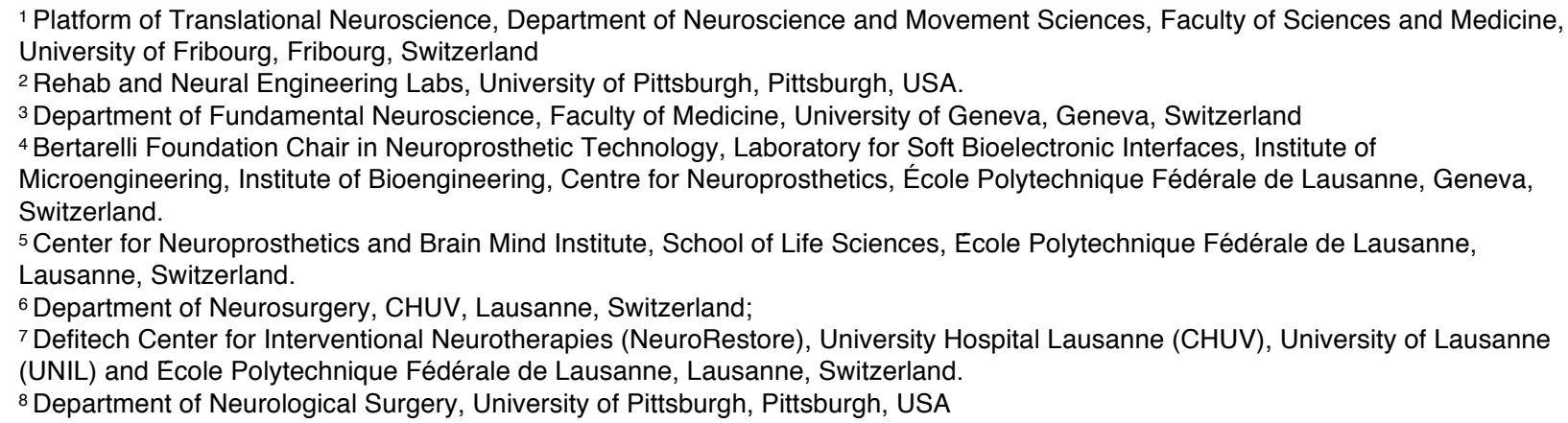

*these authors contributed equally to this work

Correspondence to: Marco Capogrosso - mcapo@pitt.edu

\section{SUMMARY}

Regaining arm motor control is critical for people with paralysis. Despite promising results on grasping, no technology could restore effective arm control. Here, we show that electrical stimulation of the cervical spinal cord enabled three monkeys with cervical spinal injury to execute functional arm movements. We designed an epidural interface that engaged surviving spinal circuits via the recruitment of large sensory afferents to produce movement. Simple stimulation bursts produced sustained joint movements which, triggered by movement-related intracortical signals, enabled monkeys with arm paralysis to perform an unconstrained, three-dimensional reach and grasp task. This restoration of voluntary motor control was enabled by the synergistic integration of spared descending commands and electrical stimulation within the spinal cord. The simplicity of this technology promises realistic clinical translation. 
More than 5 million people in the US currently live with some form of motor paralysis ${ }^{1}$. For those with impaired hand and arm control, recovery of upper limb motor function represents a top priority $^{2}$. Unfortunately, recovery of hand and arm motor function is still an unsolved clinical challenge.

Generated in the cortex, motor commands are relayed to subcortical and spinal circuits which in turn activate motoneurons to produce skilled motor actions ${ }^{3}$. Spinal cord injury (SCl), or stroke, can damage communication between these nodes leading to motor paralysis. Historically, neurotechnologies were conceived around the idea of enabling movements in paralyzed subjects via a technological bypass to extract signals from cortical areas and artificially generate muscle activity below the lesion ${ }^{4}$. For example, functional electrical stimulation (FES) directly activates arm muscles and, when coupled to intracortical brain recordings, allowed paralyzed monkeys and humans to perform skilled grasping tasks ${ }^{5-8}$. These pioneering works demonstrated the maturity of neurotechnologies as potential solutions for arm paralysis. However, translation of these systems into daily clinical practice is currently hindered by two distinct limitations. First, muscle recruitment generated by FES induces muscle fatigue ${ }^{9}$ that prevents the generation of sustained forces and consequently fails to enable three-dimensional arm movements required for daily activities. Second, since FES bypasses existing circuits, orchestrating the activation of multiple muscles to produce functional movements requires very complex stimulation protocols ${ }^{10}$ controlled by sophisticated algorithms ${ }^{6,8}$. As a result, these systems require a complex combination of hardware and software. Unfortunately, this complexity does not cope well with dynamic clinical environments that need robust and practical solutions for a rapid set up and largescale use.

In contrast, epidural electrical stimulation (EES) of the lumbar spinal cord exploits residual spinal circuits and supra-spinal connections to produce movements ${ }^{11}$ and restored weight bearing locomotion in humans with $\mathrm{SCl}$ using simple stimulation protocols and approved medical technologies ${ }^{12-14}$. Similar to intraspinal stimulation ${ }^{15-17}$, EES engages motoneurons via large sensory afferents leading to a natural motoneurons recruitment order that is resistant to artificial fatigue. This enables the production of forces that can sustain the whole-body weight ${ }^{18}$. Moreover, engagement of motoneurons from pre-synaptic pathways allows residual descending inputs and spinal circuits to control motoneurons excitability and produce voluntary movement after complete motor paralysis ${ }^{19,20}$. Enabling the amplification of residual supra-spinal inputs would be critical to restore upper limb movements with a simple technology. Therefore, translation of EES to the restoration of arm and hand movements is contingent on the ability to recruit similar sensorimotor circuits in the cervical spinal cord as in the lumbar cord ${ }^{16,21,22}$. Interestingly, spinal circuits also play a critical role in arm and hand motor control23-25, therefore we hypothesized that a neural interface, designed to target cervical sensory-motor circuits, could enable the generation of voluntary arm movements after paralysis.

Here, we tested this conjecture in monkeys with $\mathrm{SCl}$. We designed a personalized epidural interface to target primary afferents within the cervical dorsal roots. We hypothesized that the stimulation of the roots with bursts linked to movement attempts would enable voluntary motor control and improve critical functional deficits that emerge after $\mathrm{SCl}$ such as: muscle strength, dexterity to execute functional tasks, and movement quality. We tested the efficacy of our system on three adult macaque monkeys with incomplete cervical spinal cord injury. 

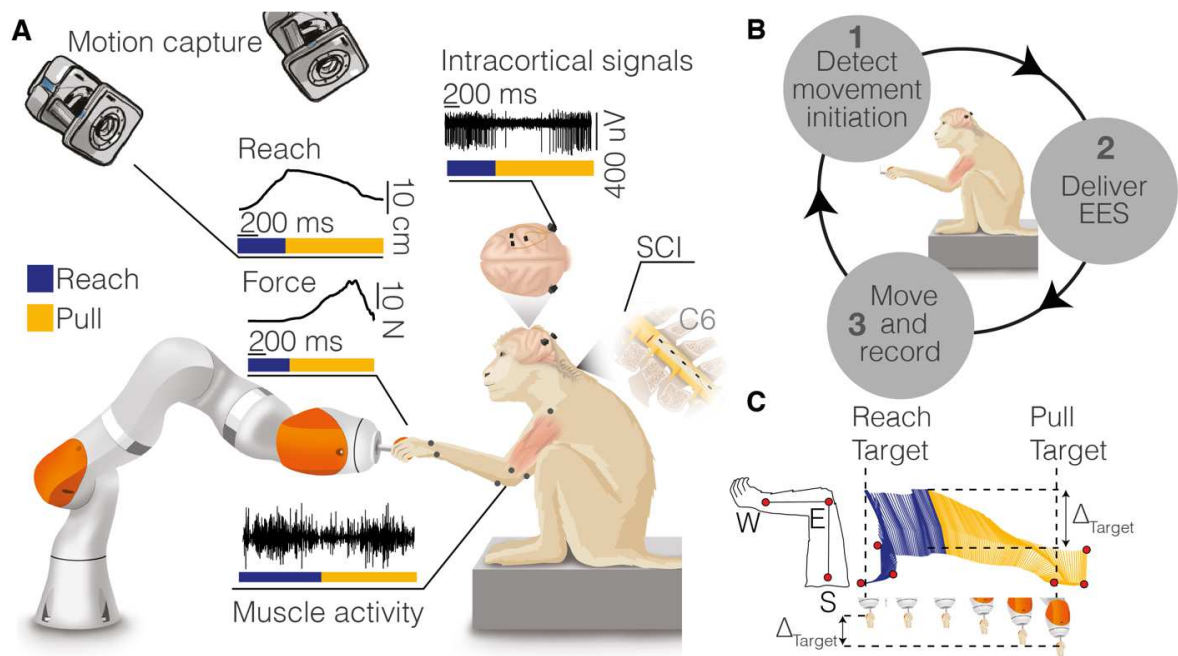

Figure 1. Experimental framework. (A) Monkeys were trained to reach for, grasp, and pull a target object placed at the end effector of a robotic arm. We measured 3D forces applied to the robot joints, full-limb kinematics, electromyographic (EMG) activity from eight muscles of the arm and hand, and intracortical signals from primary sensorimotor areas. (B) Conceptual scheme of the experimental protocol: (1) A decoder running on a control computer identified movement attempts and (2) delivered electrical spinal cord stimulation to the appropriate spinal roots. (3) Stimulation produced arm and hand movement that we recorded and analyzed off-line. (C) Stick diagram decomposition of arm movement during a reach, grasp and pull movement in intact monkeys ( $\mathrm{S}=$ shoulder, $\mathrm{E}=$ elbow, $\mathrm{W}=$ wrist). We considered a movement complete when a target spatial threshold was crossed during pull. Copyright Jemère Ruby.

\section{Results}

\section{Studying natural arm movements}

Clinically effective systems should demonstrate the ability to enable truly functional arm movements rather than simplified tasks such as single-joint movements. Consequently, we developed a robotic platform allowing the quantification of reach and grasp movements ${ }^{26}$ that would feel natural and unconstrained to monkeys. We trained three Macaca fascicularis monkeys to reach for, grasp, and pull an instrumented object placed on the end effector of a robotic arm (Figure 1). Movement trajectories were not constrained, and the monkeys intuitively and rapidly ${ }^{29,30}$ learned the task by developing their individual kinematic strategies (Extended Data Figure 1). Our system was designed to quantify functional outcomes on task performances, muscle activation, muscle strength and movement dexterity. To evaluate these outcomes, we measured full-limb 3D kinematics (Vicon Motion Systems, Oxford, UK), pulling forces, and electromyographic (EMG) signals from the principal arm muscles (Figure 1). Before the $\mathrm{SCl}$, we observed clear bursts of EMG activity throughout the upper limb in the three movement phases: reach, grasp, and pull in all monkeys. Multi-microelectrode arrays (Blackrock Microsystems, Salt Lake City, USA) implanted in the arm/hand region of the right sensorimotor (M1, S1) and premotor (PMv) cortex also showed consistent modulation of neural activity with kinematics (Figure 1, Extended Data Figure 1) as largely expected.

\section{Personalized spinal interface}

To design an optimal interface, we ascertained the anatomy of the monkey cervical spinal cord. We extrapolated available anatomical information and found that, similar to humans, motoneurons innervating arm muscles are segmentally organized ${ }^{27}$ (Figure 2A). Our previous work showed that stimulation of a single dorsal root will mainly recruit motoneurons located in the corresponding 
A

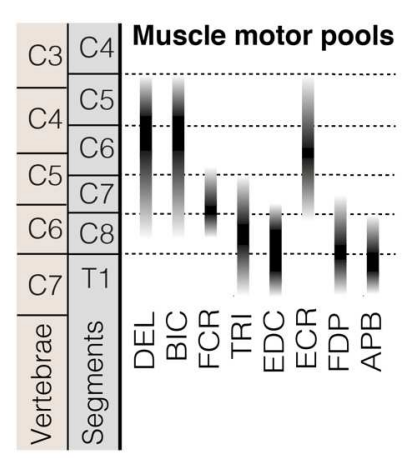

C

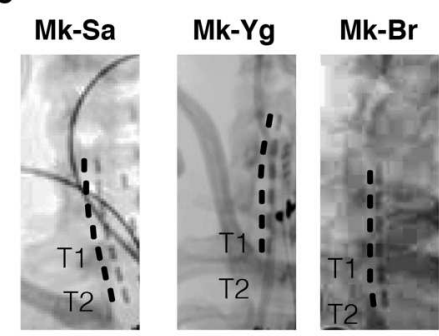

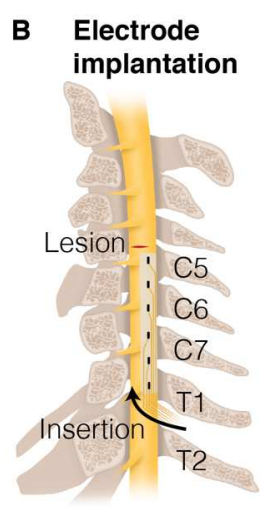

D

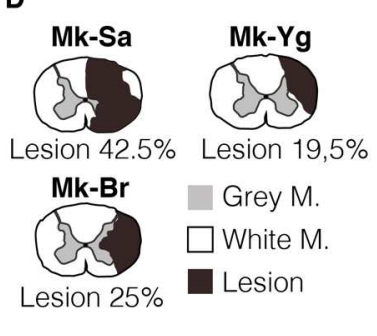

roots exit

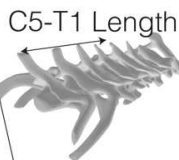

Personalization landmarks

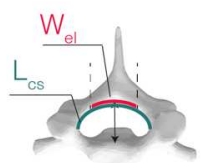

$W_{e l}=1 / 3 L_{c s}$
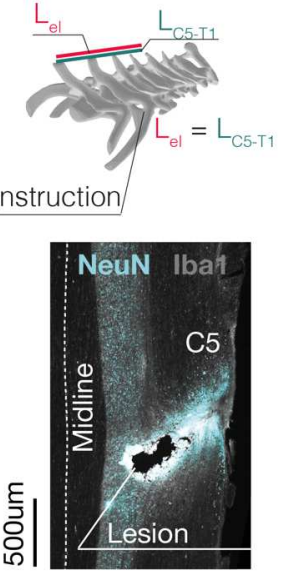

Figure 2. Epidural electrode design and implantation. (A) Motoneurons pool distribution of arm and hand muscles in the cervical spinal cord in relation to vertebrae and spinal segments (adapted from Jenny and Inukai, 1983). Deltoid (DEL), Biceps Brachii (BIC), Flexor Carpi Radialis (FCR), Triceps Brachii (TRI), Extensor Digitorium Communis (EDC), Extensor Carpi Radialis (ECR), Flexor Digitorium Profundis (FDP), Abductor Pollicis Brevis (ABP). (B) Schematic representation illustrating the positioning and insertion of the spinal implant in the epidural space; on the right, anatomical landmarks used to tailor the epidural interface to each monkey's anatomy (Length of dorsal aspect of spinal canal $L_{c s}$, length of C5-T1 spinal segment $\mathrm{L}_{\mathrm{C} 5-\mathrm{T} 1}$, electrode width $\mathrm{W}_{\mathrm{el}}$, electrode length $\left.\mathrm{L}_{\mathrm{el}}\right)$. Three-dimensional reconstructions of vertebras are obtained by CT-reconstruction (Osirix, Pixmeo, Switzerland). (C) Representative X-ray scans of the epidural implant in the three monkeys (Mk-Sa, Mk-Br and Mk-Yg). (D) Anatomical reconstruction of the cervical spinal cord lesion (black area) for the 3 monkeys, shown on a transversal section. On the right, representative image of longitudinal section of the spinal cord of $\mathrm{Mk}-\mathrm{Br}$ around the lesion site stained with NeuN (neuronal cell bodies) and Iba1 (microglia).

segment ${ }^{28,29}$. Therefore, we designed a spinal interface that could target each of the roots independently by placing contacts on the lateral aspect of the cord to target the entry zone of each individual root ${ }^{28}$. Since each monkey possessed a unique anatomy, we tailored the design of our interface to each specific subject. For this, we measured white matter diameter and vertebral canal features from Computed Tomography (CT) and Magnetic Resonance Imaging (MRI). We then spaced the electrodes rostro-caudally and medio-laterally to match the transversal and longitudinal dimensions of the cord of each animal (Figure 2B, Extended Data Figure 2A). This allowed us to simplify the neural interface architecture by minimizing the number of contacts required for whole arm muscle recruitment while maintaining high specificity and reducing the complexity of the implant ${ }^{30}$. We then designed a surgical strategy to position the epidural interface between the $\mathrm{C} 6$ and T1 dorsal roots. We performed laminectomies between the T1 and T2 vertebrae and the $\mathrm{C} 5$ and $\mathrm{C} 6$ vertebrae, then pulled the neural interface through the intermediate epidural space with the help of a custom soft inserter ${ }^{30}$. We verified that the position of the array remained stable for the entire duration of the study (up to 3 weeks) through repeated X-ray imaging (Figure 2C, Extended Data Figure 2B). During the same surgery, we performed a unilateral spinal cord injury at the C5/C6 segments (Figure 2D). Postmortem analysis showed 
reveal that $\mathrm{Mk}-\mathrm{Br}$ received an unplanned compression injury at the insertion site (T3 spinal segment), which may have occurred during implantation (Extended Data Figure 2C). Since the T3 segment is below the innervation of the arm motoneurons, this lesion did not affect the phenotype of arm and hand deficits that did not differ from the other monkeys (See Methods).

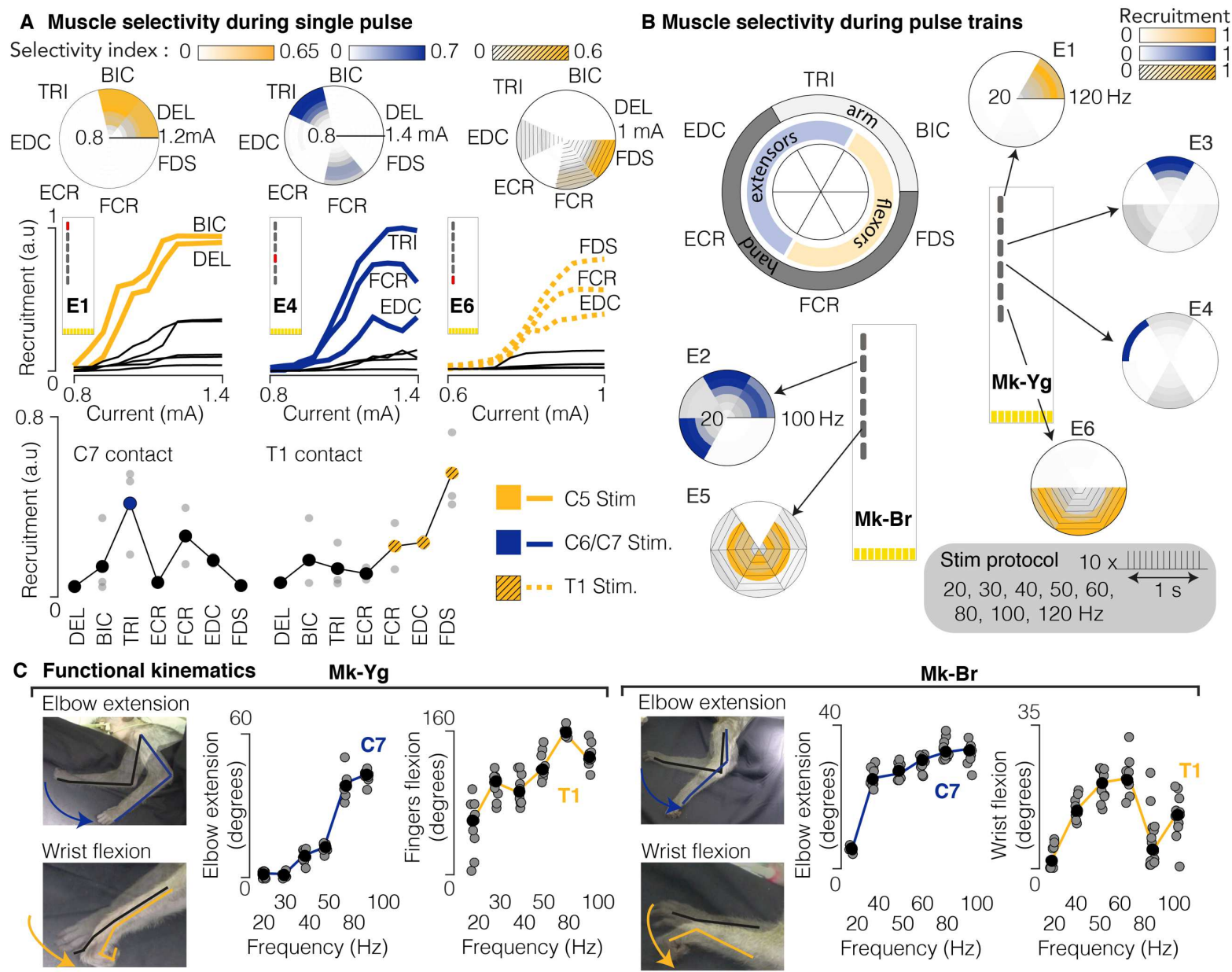

Figure 3. EES produces single joint movements in anesthetized animals. (A) Examples of muscle selectivity (polar plot) and muscle recruitment obtained by stimulating $(1 \mathrm{~Hz})$ at $\mathrm{C} 5, \mathrm{C} 6 / \mathrm{C} 7$, and T1 spinal segments (Mk-Yg). Below, average muscle activations elicited from $\mathrm{C} 7$ and T1 contacts in $\mathrm{n}=3$ monkeys (Grey bullets: for each animal, average recruitment across all stimulation currents. Big bullets: mean of average recruitments across animals). (B) Muscle recruitment obtained during delivery of pulse trains in anesthetized monkeys. Recruitment was estimated by computing the energy of EMG signals for each muscle and each stimulation contact. Stimulation frequencies ranged from 20 to $120 \mathrm{~Hz}(n=2)$. For each muscle, energy values were normalized to the maximum value obtained across all frequencies and contacts. (C) Single joint angles excursions induced by stimulation at C7 (blue) and T1 (yellow) roots. Stimulation frequencies ranged from 20 to $100 \mathrm{~Hz}(n=2)$. Black bullets: mean. Line: interpolation of the mean values.

\section{Cervical EES produces single joint movements}

132 We next assessed the selectivity of the epidural interface. In propofol anaesthetized monkeys, we delivered asymmetric, charge-balanced biphasic pulses of EES at low repetition rate $(1 \mathrm{~Hz})$ at various current amplitudes from each contact. Minimum and maximum amplitude values were selected as the first subthreshold and first saturation current value respectively. As predicted ${ }^{28}$, 
different stimulation contacts generated muscle recruitment patterns that mirrored the segmental organization of cervical motoneurons (Figure 3A, Extended Data Figure 3A). Specifically, caudal contacts elicited spinal reflexes mostly in the hand and forearm muscles, while rostral contacts recruited biceps and deltoids.

To ensure that this segmental selectivity translated into functional arm and hand movements, we delivered supra-threshold stimulation at various frequencies $(20-120 \mathrm{~Hz})$ from each contact in two animals ( $\mathrm{Mk}-\mathrm{Br}$ and $\mathrm{Mk}-\mathrm{Yg}$ ). Selectivity was preserved during long stimulation trains (Figure 3B) and different contacts elicited distinct joint movements (Video 1). For example, contacts primarily targeting the $\mathrm{C} 7$ root (innervating triceps) produced clear elbow extension; instead, caudal contacts (C8/T1) elicited grasping and wrist movements (Figure 3C, Extended Data Figure 4). All single joint angles excursions were gradually modulated by varying the stimulation frequency (Figure 3C). In most of the upper arm muscles we found a monotonic relationship between muscle activation and stimulation frequency. However, for some muscles (e.g. abductor pollicis), responses were lower at higher frequencies (Extended Data Figure 3B). We identified the optimal stimulation range to be around $50-60 \mathrm{~Hz}$ (Figure 4). Movements elicited at frequencies lower than $40 \mathrm{~Hz}$ were often too weak to complete a joint movement; bursts at frequencies between 50 and $60 \mathrm{~Hz}$ produced smooth ${ }^{31}$ and full-range movements and maximal forces, while frequencies higher than $60 \mathrm{~Hz}$ produced either abrupt movements or incomplete movements (Figure 4A) due to attenuation of muscle responses during stimulation of sensory afferents $28,32,33$ (Extended Data Figure 4B). We identified three stimulation contacts that could consistently elicit arm extension (reach), hand flexion (grasp) and arm flexion (pull) (Figure 4B). We then verified that this selection of few contacts could be used to sustain reaching, grasping and pulling movements. By sequentially executing bursts on these three contacts, we could trigger whole arm movements that mimicked smooth ${ }^{31}$ and natural multi-joints movements (Figure 4C, Video 1). Extension, grasping and pulling movements produced clear EMG bursts as well as robust and smooth kinematics. These data demonstrate that with only three contacts, stimulation bursts can engage functionally relevant muscles that produce whole arm movements and sustained muscle activation and forces. Therefore, we planned to link the delivery of these bursts to movement onsets that we derived from intra-cortical signals. Indeed, since our lesions were not complete, movement onsets could be reliably detected even after $\mathrm{SCl}$ from intra-cortical signals (Figure 4D). Similarly to other spinal cord stimulation studies we could not identify contacts that selectively produced finger extension ${ }^{34}$. This is likely caused by the overlap of extensor motor-pools in the forearm ${ }^{27}$ (Figure 2A), but possibly also because stronger flexors may dominate kinematics in the case of co-contraction at rest.

\section{EES improves arm control after spinal cord injury}

We next tested whether our stimulation protocol could improve functional outcomes of upper limb movements. Specifically, we tested the efficacy of EES to improve muscle activation, pulling forces, functional task performance, and kinematic quality of three-dimensional movements after $\mathrm{SCl}$. In all monkeys, the unilateral lesion led to motor deficits of the left arm and hand. Each monkey retained the ability to activate proximal shoulder and biceps muscles, while elbow and hand function were compromised. Severity of the impairment and extent of spontaneous recovery (Extended Data Figure 5B) varied across monkeys because of the variability in lesion size (Figure 2D). Generally, animals showed severe paralysis immediately after lesion, and then gradually regained some movement capabilities (Extended Data Figure 5B). Due to the initial impairment, immediately after the lesion, monkeys were not able to perform the behavioral task. Consequently, during the first week, we simplified the task by presenting an object close to the 


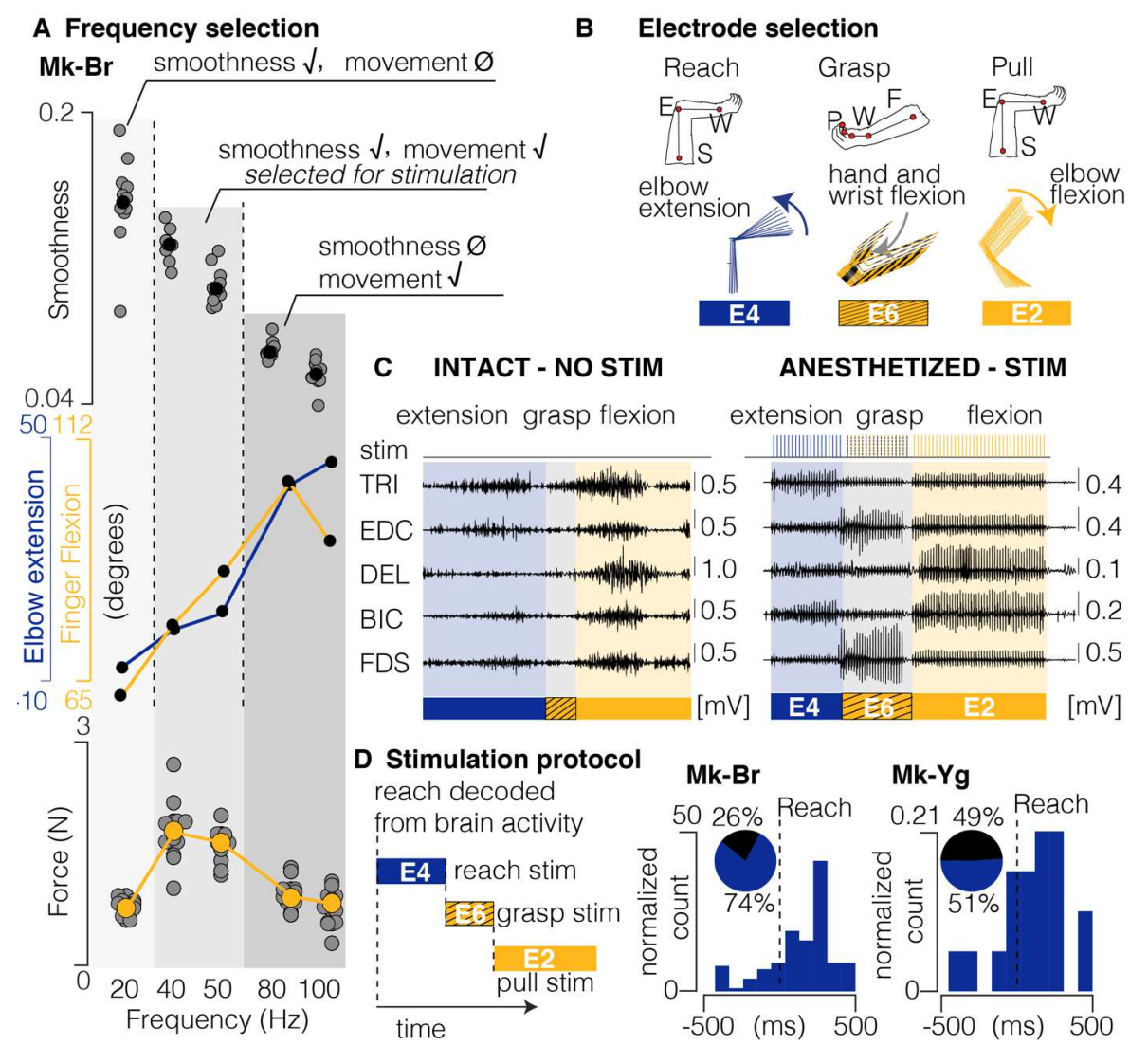

Figure 4. Design of stimulation protocol. (A) Combined representation of movement smoothness, elbow and finger flexion, and pulling force during anesthetized stimulation. Shades of gray highlight three frequency ranges that produce: (1) smooth trajectory, but little movement and low force $(2 \mathrm{~Hz})$, (2) smooth trajectory, extended movement and medium force $(40$ and $50 \mathrm{~Hz}),(3)$ abrupt and very extended movement and low force $(80$ and $100 \mathrm{~Hz})$. The range $40-50 \mathrm{~Hz}$ was selected as the best optimization of sufficient movement, smoothness and force production. (B) Schematic representation of arm and hand kinematics during stimulation delivered from the selection of three contacts to produce elbow extension (blue), hand and wrist flexion (yellow and black), and elbow flexion (yellow). (C) Example of comparison between EMG activity during intact movement (left) and movement elicited by chaining stimulation from the three selected contacts (right). (D) Scheme illustrating how stimulation is triggered from movement-related intra-cortical signals. On the right, online performances of movement attempt decoder in two animals with SCl. Pie charts represent percentage of predicted (blue) and unpredicted (black) reach events by our decoder.

monkeys and triggering stimulation manually to encourage the animal to perform the task. After the first week, all monkeys spontaneously attempted to perform the task, making it possible to link the delivery of stimulation bursts to real-time detection of movement onset using intra-cortical signals. Whenever the monkeys strived for a reach, grasp or pull movement, we delivered bursts of stimulation promoting reach or grasp/pull respectively. Outcomes were computed for each animal independently and compared between EES on, and EES off conditions. EES significantly enhanced muscles activity and forces (Figure 5B,D) compared to no stimulation. In terms of functional task performances, without stimulation, the monkeys were rarely capable of completing any part of the task (defined as reach, grasp and pull). Instead, with the support of EES, the rate of successes was significantly and robustly improved (Figure 5C, Video 2,3,4). EES did not only improve task performance and strength but also overall quality of movement (Figure 5D). Indeed, principal component analysis (PCA) of three-dimensional kinematic parameters (i.e., timing, force, 
A

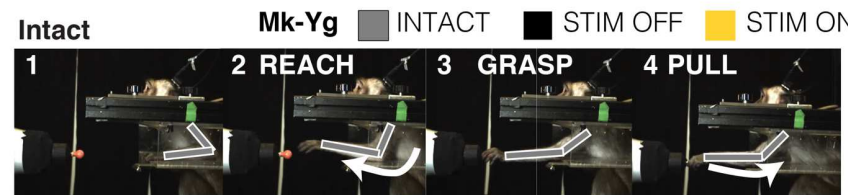

After SCl - stim OFF

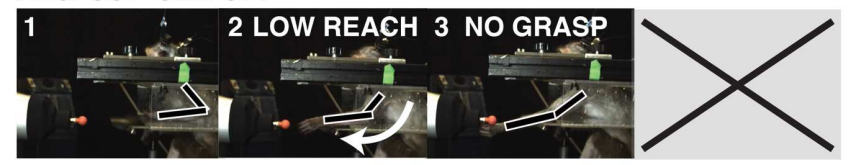

After $\mathrm{SCl}$ - stim ON

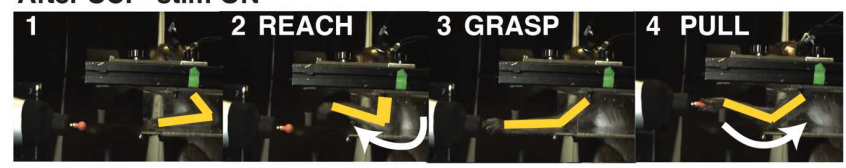

C EES improves task performance

Mk-Br, week 3

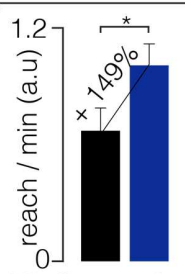

Mk-Sa, week 1

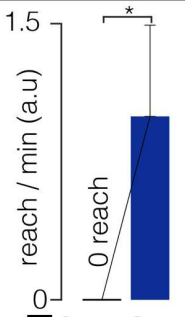

STIM OFF
1.27 **
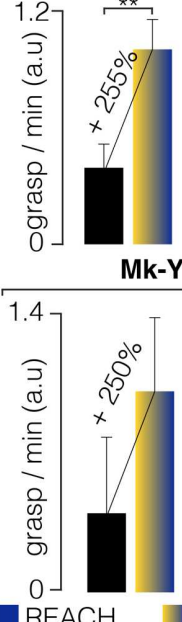

Mk-Yg, week 2
D EES improves kinematics
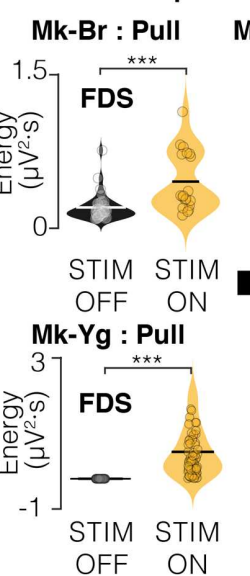

Mk-Br STIM OFF STIM ON

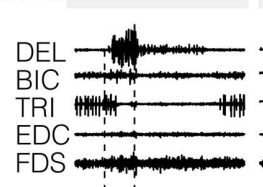

sta'rt igrasp

STIM OFF —REACH STIM PULL STIM
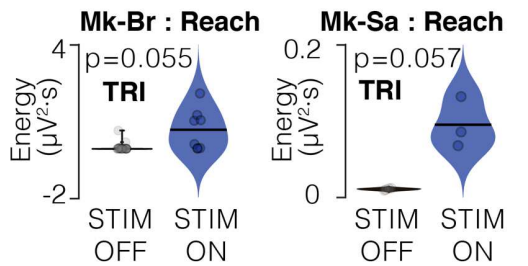

Mk-Yg

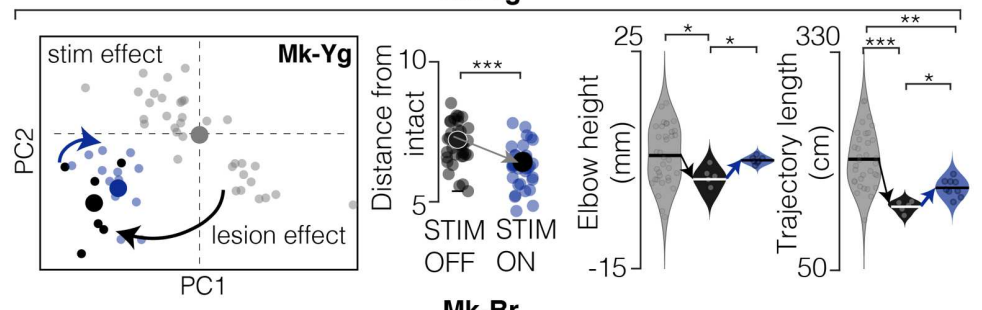

Mk-Br

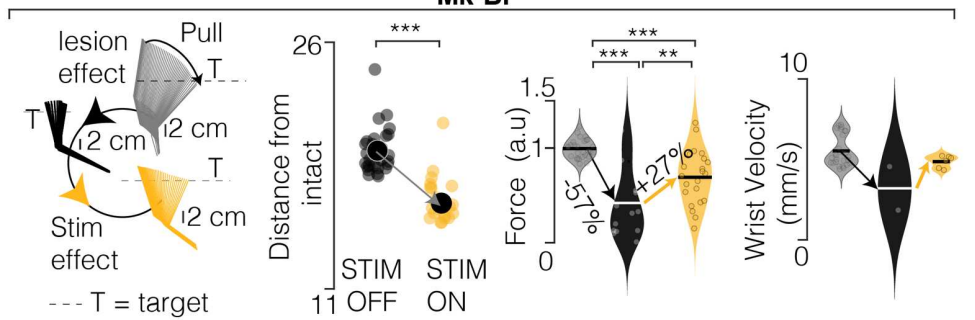

Figure 5. EES improves task performance, muscle strength and movement quality. (A) Snapshots of Mk-Yg performing the task before $\mathrm{SCl}$, after $\mathrm{SCl}$ without $\mathrm{EES}$, and after $\mathrm{SCl}$ with $\mathrm{EES}$. A full successful trial is composed of a reach, a grasp, and a pull. After SCl, Mk-Yg could only perform reaching movements without EES, while when EES was delivered the full task could be performed. (B) Violin plots of signal energy of triceps and FDS EMG profiles during reach ( $\mathrm{Mk}-\mathrm{Br}$ and $\mathrm{Mk}-\mathrm{Sa}$ ) and pull ( $\mathrm{Mk}-\mathrm{Br}$ and $\mathrm{Mk}-\mathrm{Yg}$ ). All individual data points are represented by bullets. Black lines correspond to means of the distribution. Statistical analysis with Wilcoxon Ranksum test. On the right, example raw EMG data after SCI with and without EES. (C) Bar plots report the rate of successful movements after $\mathrm{SCl}$, without and with stimulation. Data are presented as mean \pm STD and normalized on the mean value in stimulation condition. Statistics was performed with Bootstrap. (D) Example PC analysis of kinematic features (See methods). Top-left, first and second PC space. Bottom left, stick diagram representation of arm kinematics during pull in intact conditions, after SCl without and with EES. At the immediate right (both bottom and top), euclidean distance in the feature space of trials without stimulation (black) and with stimulation (blue) from the centroid of the trials in intact condition. At the extreme right, example violin plots of movement quality features in the three conditions: intact, after $\mathrm{SCl}$, and after $\mathrm{SCl}$ with stimulation. Statistics with Wilcoxon Ranksum test. Asterisks: ${ }^{*} p<0.05,{ }^{* *} p<0.01,{ }^{* * *} p<0.001$.

arm trajectories, joint angles) revealed that during EES, movement kinematics were significantly 

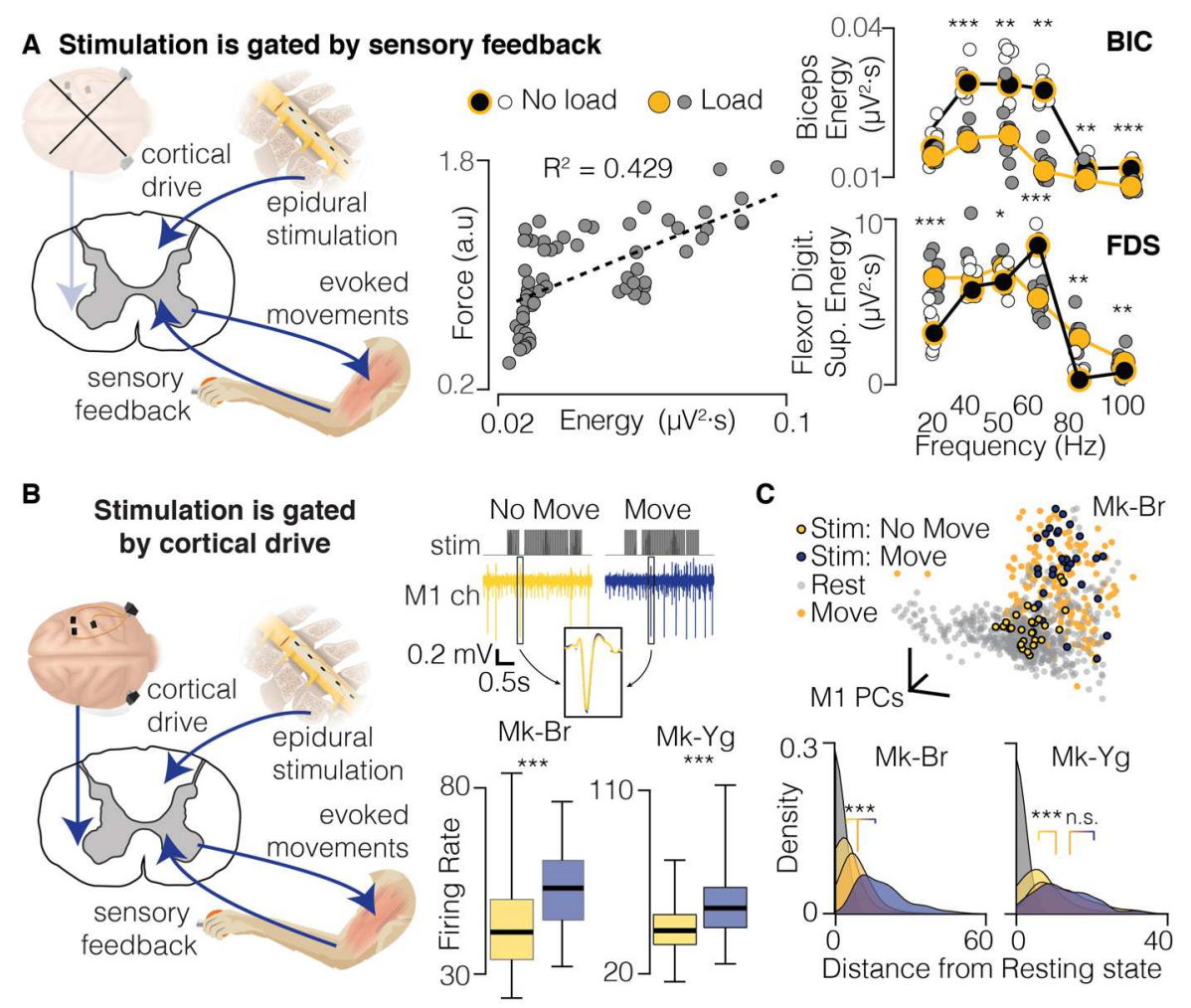

Figure 6. EES must be synchronized with motor intention. (A) Left: EES modulates spinal circuitry to presynaptically recruit motoneurons innervating the muscles of the arm. Thus, EES interacts with descending cortical drive sent through residual pathways after SCl. Middle: correlation between energy of the EMG trace of the biceps muscle and arm flexion force produced by muscle contraction during a isometric arm flexion induced by stimulation. Right: energy of EMG signal of biceps and FDS muscles during free arm flexion (no load) or isometric arm flexion (load) induced by stimulation. White and grey bullets: individual data points for no load and load conditions. Black and yellow bullets: mean values for no load and load conditions. Black and yellow lines: interpolation of mean values for no load and load conditions. (B) (Top) The same EES pulse train (top) applied to $\mathrm{Mk}-\mathrm{Br}$ can result in different motor output. For an example M1 channel, the stimulation that evoked movement (blue, right) corresponded to more spiking activity than the same stimulation evoking no movement (yellow, left). (Bottom) Distribution of average firing rates across all M1 channels during stimulation trains that evoked no movement (yellow) and movement (blue). (C) (Top) State space view of M1 activity for all time points during rest (gray) and preceding attempted movement (orange). The brain states during successful stimulation (blue) were similar to those preceding attempted movements, while the unsuccessful stimulation (yellow) overlapped with the rest states. (Bottom) We computed a relative Mahalanobis distance between the two stimulation conditions and the cluster of neural states at rest. For both monkeys, neural states during stimulation periods with no movement were close to rest.

performed wider movements, and generated stronger forces (Figure 5D), getting closer to normal kinematic trajectory patterns without any long-term training.

\section{Sensory feedback and cortical inputs shape EES efficacy.} We then investigated the role of spinal circuits and residual cortical inputs in the regaining of voluntary movements that we observed. Indeed, since activation of motoneurons was presynaptic, both spinal reflexes and residual cortical inputs could shape motor output during EES ${ }^{19,35}$. First, we assessed the influence of sensory inputs on EES-generated motor output. Under propofol anesthesia, we delivered bursts of EES targeting the elbow flexion in isometric conditions (Figure 6A). We found that induced EMG activity was highly correlated with measured 
210 force output. We then performed the same experiments under unconstrained kinematics and 211 found that EMG activity for different EES frequencies was significantly different from those of 212 isometric movements (Figure 6A). The force load at the hand changed the input/output 213 relationship between EES stimulation frequency and EMG activation. Under anesthesia, only changes in sensory feedback can explain the observed changes on EES motor effects.

Second, we noticed that, at the start of the task, during false positive movement detections, despite EES was delivered the monkeys did not move or performed the task. Instead, if stimulation bursts were delivered during movement but at a wrong time, movements could be induced and even impair task execution (Video 4, part 3). We then hypothesized that, when awake, residual supra-spinal inputs needed to be in a movement-permissive state to enable voluntary movements with EES (Figure 6B). To test this hypothesis, we examined post-hoc neural spiking activity from the primary motor cortex (M1) of $\mathrm{Mk}-\mathrm{Br}$ and $\mathrm{Mk}-\mathrm{Yg}$ during true positive and false positive trials. We identified trials where EES enhanced muscle activation and compared it to events where EES did not generated any muscle activity in relation to M1 activity at rest or during movements without stimulation. We found that motor cortex was significantly more active when EES produced movement (Figure 6B) than when it did not. We then applied PC analysis to reduce the M1 population activity to low-dimensional states and compare M1 activity during EES with periods of no stimulation (Figure 5C). Interestingly, during false positive stimulation resulting in no motor output, overall M1 neural activity was closer to activity at rest. Instead, when stimulation resulted in successful muscle activation, M1 neural activity overlapped with activity observed during movement states with no stimulation. These results are in agreement with our hypothesis that volitional cortical input was necessary to enable the production of effective movements during EES.

\section{Discussion}

We showed that EES of cervical spinal cord immediately improved muscle activation and strength, task performances and movement quality during a natural-like reach and grasp task in monkeys with unilateral cervical SCI. Moreover, these results were obtained with simple stimulation protocols engaging up to three contacts (one for reach, one for grasp and one for pull) that enabled multi-joint movements. We believe that the design of our interface was key to achieve this result. The dorsal roots are a robust anatomical target that we could easily identify through standard imaging to personalize surgical planning and interface design. Our simple protocol only required the detection of movement onset signals to trigger pre-determined stimulation bursts. Therefore, stimulation control could be simplified and brain recordings may not be required in clinical applications that might exploit more practical residual movements in patients with incomplete paralysis ${ }^{14,36}$.

By engaging spinal circuits, EES generated smooth and functional muscle activations that enabled the production of forces sustaining the weight of the arm. Moreover, EES was sensitive to the action of residual descending cortical inputs allowing the cortex to shape voluntary muscle activation and inhibition to produce a desired kinematic output ${ }^{37,38}$. Indeed, the analysis of brain data during voluntary execution of moments with EES suggested that the cortex must be in a movement-permissive state to enable movement with EES. Indeed, in order to produce a functionally relevant motor output, stimulation bursts had to be coherent to motor intention. These features might be regarded as limitations: activating muscles with segmental specificity implies the impossibility to achieve single-muscle recruitment, and the sensitivity and dependence on residual cortical inputs implies a potential failure of EES in motor complete injuries. However, previous studies showed that even completely paralyzed subjects retain residual but functionally 
silent descending inputs ${ }^{12,14,19}$. Therefore, residual cortical activity may help shaping EES efficacy even in severe patients. In summary, we believe that by exploiting the functionality of residual spinal circuits and supra-spinal inputs EES constitutes a simple yet robust approach to the restoration of arm motor control with high translational potential.

\section{Acknowledgements}

The authors would like to thank Jacques Maillard and Laurent Bossy for the care provided to the animals, Dr Eric Schmidlin and Simon Borgognon for their help with anaesthesia and surgery preparations, Marion Badi for her help and advice during experiment preparations and experimental procedures, Dr. Andrina Zbinden for her contribution to the health survey of the monkeys, Andre Gaillard and Andrea Francovich for their help with the implementation of the hardware and the students of the University of Fribourg Amélie Jeanneret, Alen Jelusic, Laora Marie Jacquemet and Samia Borra for their help in processing data.

\section{Funding}

The authors would like to acknowledge the financial support from the Wyss Center grant (WCP 008) to MC, GC and TM, an industrial grant from GTX medicals to GC and MC; the Bertarelli Foundation (Catalyst Fund Grant to MC and TM and funds to SL) a Swiss National Science Foundation Ambizione Fellowship (No. 167912 to MC), The European Union's Horizon 2020 research and innovation program under the Marie Skłodowska-Curie grant agreement no. 665667 (GS) the Swiss National foundation grant BSCGI0_157800 (SL), a Whitaker International Scholars Program fellowship to MGP, and an internal pilot grant of the University of Fribourg to MC.

\section{Author Contributions}

MC, BB and SC conceived the study; BB, MGP, and TM designed and implemented the hardware and software tools; SC designed the behavioral task and training strategy; GS and SL designed and manufactured the implantable interface; BB, SC, MGP and MC conducted the experiments; $\mathrm{BB}, \mathrm{SC}, \mathrm{MGP}$ and $\mathrm{KZ}$ performed the data analysis; SC, MD and MK trained the animals; SC, KG, $\mathrm{NJ}$ and $\mathrm{QB}$ processed the histological data; JB, GC and $\mathrm{MC}$ designed surgical implantation strategies and stimulation strategies. GC and JB, performed surgical implantations and lesions. EMR and MC implemented and supervised procedures on monkeys; MC, BB, SC and MGP wrote the manuscript; all authors edited the manuscript; SL, TM, JB, GC and MC secured funding for the study; MC supervised the study.

\section{Competing Interests}

G.C., J.B., S.L., M.C., B.B. and K.Z. hold various patents in relation to the present work. G.C., S.L. and J.B. are founders and shareholders of GTX medical, a company developing an EESbased therapy to restore movement after spinal cord injury.

Data and materials availability

All software and data will be available upon reasonable request to the corresponding author. 
A Monkey's specific movement performance

B Kinematic strategy
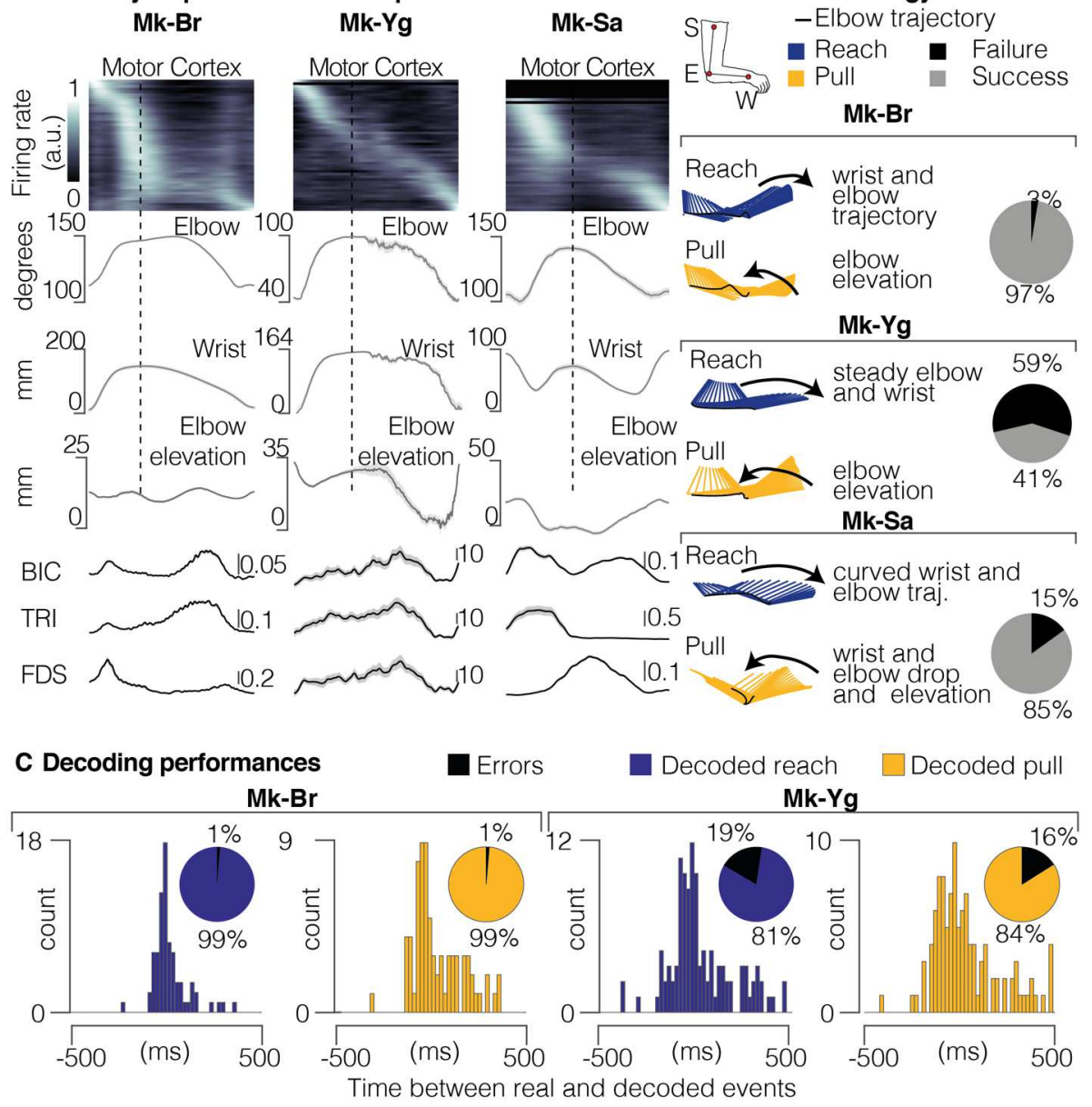

Extended Data Figure 1. (A) Portfolio of signals recorded during intact movement for each animal. These signals have been recorded during the experimental session prior to the lesion. Black line corresponds to the mean profile across all trials, shaded area shows the SEM across all trials. (B) Kinematic strategies implemented by each monkey. Stick diagrams representations of the arm kinematic during reach (blue) and pull (yellow). The black line highlights the elbow trajectory. Pie charts represent the percentage of success and failure in task performance before lesion. (C) Offline decoding performance for Mk$\mathrm{Br}$ and $\mathrm{Mk}-\mathrm{Yg}$ before lesion. Histograms show the timing accuracy of detected reach (blue) and grasp (yellow) events. Pie charts (inset) show the percentage of correctly identified events. 
A

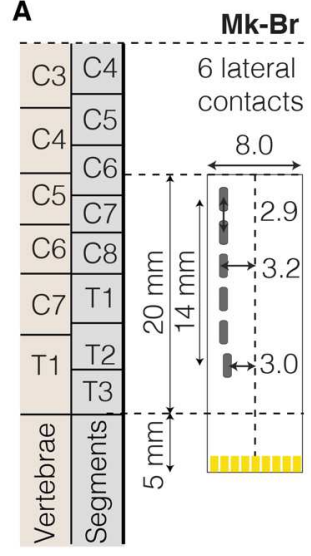

Mk-Yg

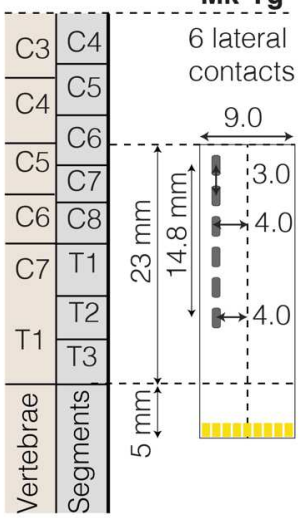

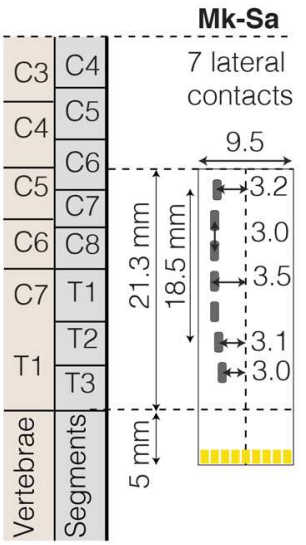

B Position stability of the implant over time C
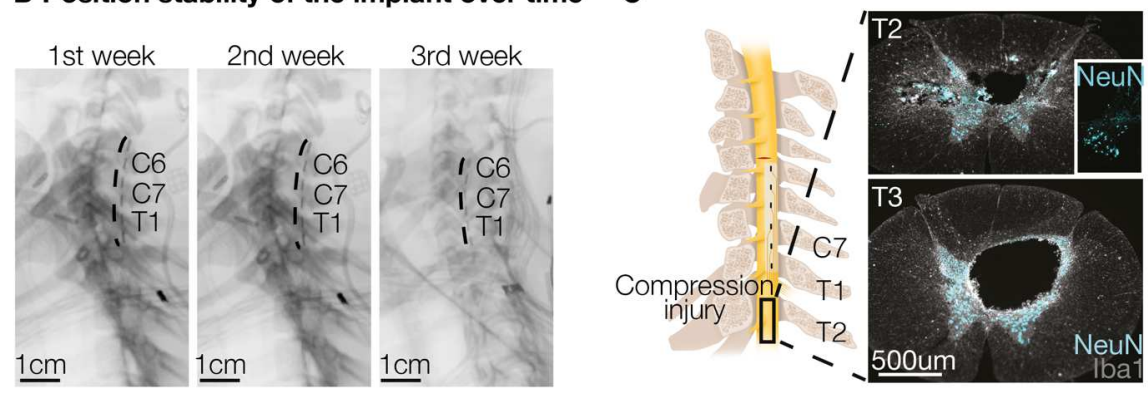

Extended Data Figure 2. (A) Personalized design of the epidural implant for each animal. All measures are in millimeters. Yellow traces at the bottom of the electrode identify connectors. (B) Position stability of the epidural array over time, illustrated through Xrays imaging taken during 3 consecutive weeks after the implantation. (C) Compression injury at the insertion level of the array (T2-T3 segment) in $\mathrm{Mk}-\mathrm{Br}$, discovered postmortem, stained with NeuN and Iba1. 


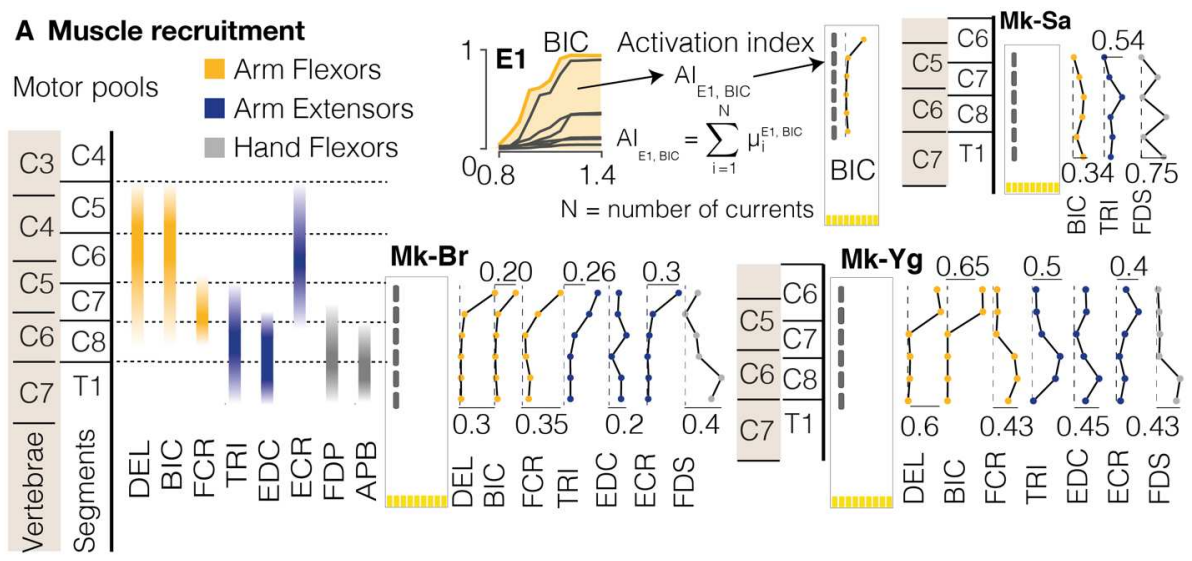

B Graded muscle activation during train pulses

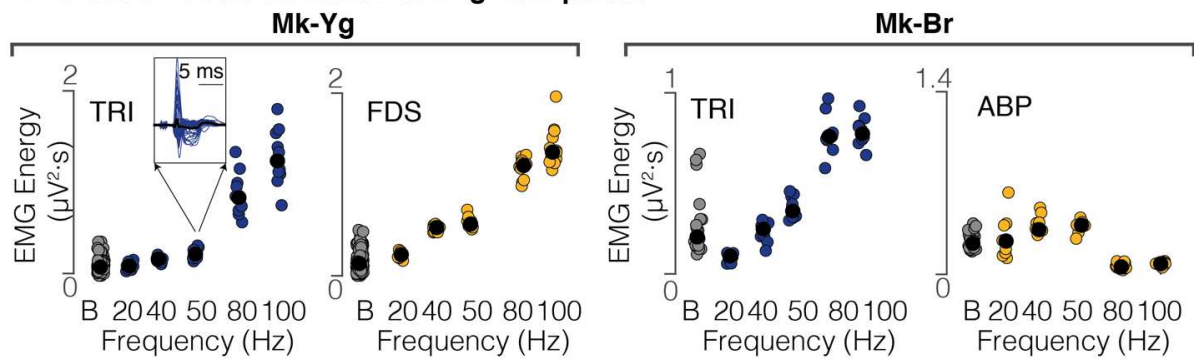

C Muscle responses are modulated at higher frequencies

Mk-Yg

Mk-Br

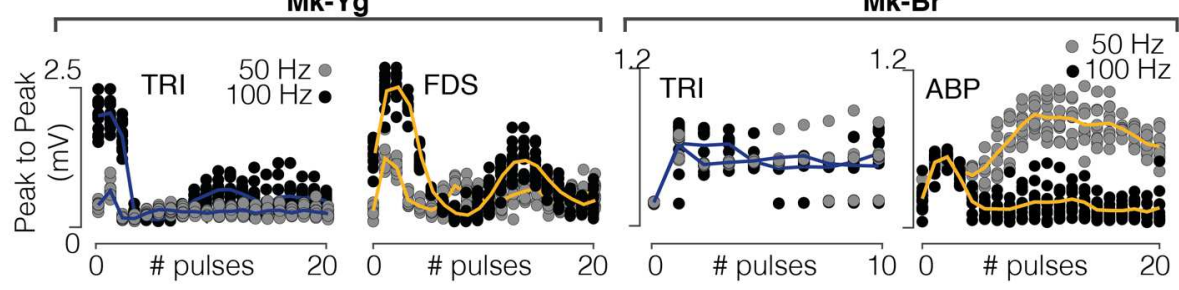

Extended Data Figure 3. (A) Single pulse muscle recruitment for each animal, contact, and muscle. Bullets identify the Activation Index (computation illustrated in the schematic above). Each bullet corresponds to a specific muscle (on the x-axis) and a specific contact (on the $y$-axis, illustrated in the implant schematic on the left). Lines connect bullets corresponding to the same muscle, across different stimulation contacts. (B) Energy of EMG signals of triceps (Mk-Br and Mk-Yg), Flexor Digitorium Superficialis (Mk-Yg) and abductor pollicis (Mk-Br) muscles, following pulse-train stimulation at different frequencies (on the X-axis). Black bullets represent mean values. (C) Evolution over time of the peak to peak value of stimulation evoked responses during a stimulation burst. Each plot shows the evolution for a specific muscle following pulse-train stimulation at 50 and $100 \mathrm{~Hz}$. Triceps is shown for $\mathrm{Mk}-\mathrm{Br}$ and $\mathrm{Mk}-\mathrm{Yg}$, Flexor Digitorium Superficialis for Mk$\mathrm{Yg}$ and abductor pollicis for Mk-Br. Each data point is represented by a bullet and lines represent mean values over time. 


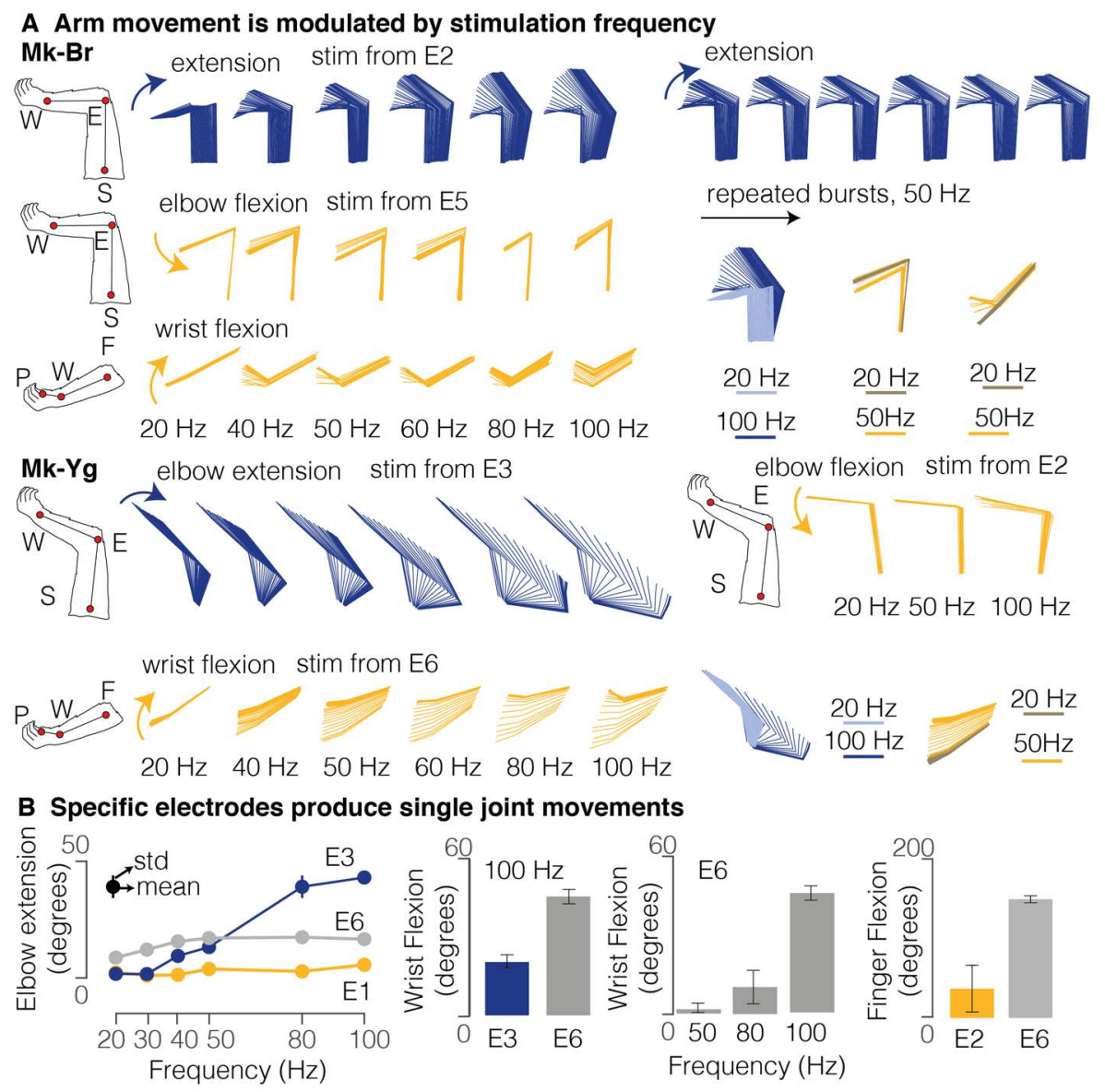

Extended Data Figure 4. (A) Stick diagram schematic of movements elicited by pulsetrains of stimulation in anesthetized conditions. Mk-Br: on the left, arm kinematic obtained by delivering stimulation at different frequencies from contacts number 2 and 5 (counting from the top); on the top-right, arm kinematics obtained by repetitive delivery of a burst at $50 \mathrm{~Hz}$; on the bottom right, superimposition of stick diagrams obtained with stimulation at $20 \mathrm{~Hz}$ and at higher frequencies ( 50 or $100 \mathrm{~Hz}$ ). For Mk-Yg: arm kinematic obtained by delivering stimulation at different frequencies from contacts number 3 and 6 and superimposition of stick diagrams obtained with stimulation at $20 \mathrm{~Hz}$ and at higher frequencies (50 or $100 \mathrm{~Hz}$ ). (B) On the left, elbow extension produced by stimulation at different frequencies. Bullets represent the mean value across different pulse-trains, and lines represent the standard deviation. Note that most of times standard deviation is so small that it remains hidden from the bullet. At the immediate right, wrist flexion obtained by stimulation through different contacts (at $100 \mathrm{~Hz}$ ) and at different frequencies (from contact number 6). At the extreme right, wrist flexion obtained by stimulation through different contacts. Values are plotted as the mean \pm STD. 

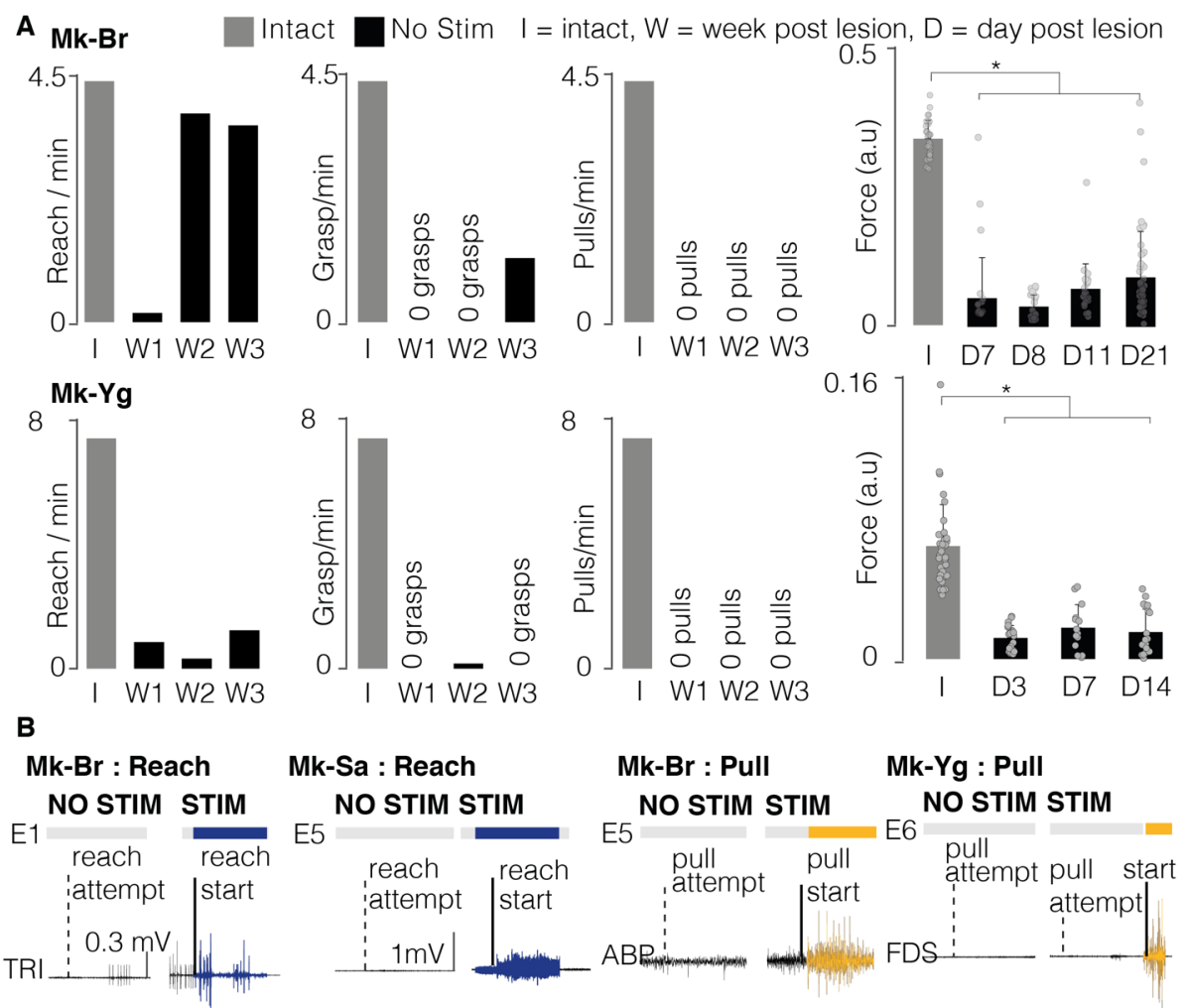

Extended Data Figure 5. (A) Illustrations of Mk-Sa and Mk-Br performing the task before $\mathrm{SCl}$ and after SCl without and with EES. A full successful trial is composed of a reach, a grasp and a pull. After SCl, Mk-Sa could not perform any movement, while when EES was delivered she could perform a reach movement. After $\mathrm{SCl}, \mathrm{Mk}-\mathrm{Br}$ could perform a weak reach and grasp but could not perform a pull, while when EES was delivered she could perform the complete task. (B) Evolution (in days) of pull force after SCl with and without stimulation. Values are plotted as the mean \pm SEM. Statistical analysis was carried out with Wilcoxon Ranksum test. (C) Examples of enhancement of EMG activity produced by EES after $\mathrm{SCl}$ in the three animals. 


\section{Animals involved in the study}

All procedures were carried out in accordance to the Guide for Care and Use of Laboratory Animals ${ }^{39}$ and the principle of the $3 R$ s. Protocols were approved by local veterinary authorities of the Canton of Fribourg (veterinary authorization No 2017_04E_FR), including the ethical assessment by the local (cantonal) Survey Committee on Animal Experimentation and final acceptance by the Federal Veterinary Office (BVET, Bern, Switzerland). Three adult female Macaca Fascicularis monkeys were involved in the study (Mk-Sa 9 years old, $4.0 \mathrm{~kg}, \mathrm{Mk}-\mathrm{Br} 3$ years old, $3.4 \mathrm{~kg}, \mathrm{Mk}-\mathrm{Yg} 3$ years old, $4.0 \mathrm{~kg}$ ). Animals were not food deprived, could freely access water at any time and were housed in collective rooms designed in accordance to the Swiss guidelines (detention in groups of 2-5 animals in a room of $45 \mathrm{~m}^{3}$ ). Rooms were enriched with toys, food puzzles, tree branches and devices to climb and hide, as well as access to an outdoor space of 10-12 $\mathrm{m}^{3}$ ). Detailed information on which animals were involved in specific experimental procedures are reported in Supplementary Table 1.

\section{Surgical procedures}

For each animal, we performed three surgical procedures, (1) intracortical electrodes implantation, (2) intramuscular electrodes implantation, and (3) epidural implant insertion and spinal cord injury. Mk-Sa deviated from this protocol. Mk-Sa was first implanted with the epidural interface before injury, however an infection occurred and resulted in the explanation of the lead to treat the infection. After recovery, the animal was re-implanted, and lesion performed following the same protocol of $\mathrm{Mk}-\mathrm{Br}$ and $\mathrm{Mk}-\mathrm{Yg}$. All the surgical procedures were performed under full anaesthesia induced with midazolam ( $0.1 \mathrm{mg} / \mathrm{kg}$, i.m.), methadone $(0.2 \mathrm{mg} / \mathrm{kg}$, i.m.), and ketamine $(10 \mathrm{mg} / \mathrm{kg}$, i.m.) and maintained under continuous intravenous infusion of propofol $(5 \mathrm{ml} / \mathrm{kg} / \mathrm{h})$ and fentanyl $(0.2-1.7 \mathrm{ml} / \mathrm{kg} / \mathrm{h}$ ) using standard aseptic techniques. A certified neurosurgeon (Dr. Jocelyne Bloch, CHUV, Lausanne, Switzerland) performed all the surgical procedures.

During the first surgical procedure, we implanted multi-microelectrode arrays in the primary motor cortex (M1-42 channels), ventral premotor cortex (PMv-32 channels) and sensory cortex (S1-42 channels) for a total of 128 channels for Mk-Br and Mk-Yg (Blackrock Microsystems, $400 \mu \mathrm{m}$ pitch and electrodes tip lengths $1.5 \mathrm{~mm} 1.5 \mathrm{~mm}$ and $1 \mathrm{~mm}$ for $\mathrm{M} 1$, PMv and S1 respectively). Instead, Mk-Sa was implanted with 2 microelectrode arrays of 64 channels each and pitch of 1.5 and 1 $\mathrm{mm}$ in $\mathrm{M} 1$ and PMd respectively. Functional motor areas of the arm were identified through anatomical landmarks and intra-surgical micro-stimulation. In order to access the brain areas of interest we performed a $20 \mathrm{~mm}$ diameter craniotomy and we incised the dura. The arrays implantation was achieved using a pneumatic compressor system (Impactor System, Blackrock Microsystems). A pedestal (Pedestal A) was then fixated to a compliant titanium mesh (Medtronic Ti-Mesh) modelled to fit the skull shape and implanted in a previous surgery a few weeks earlier ${ }^{26}$.

During the second surgical procedure we implanted intramuscular electrodes (Teflon-coated stainless-steel wires, Cooner Wire, cat. no. AS631). Mk-Yg received electrodes in the following 
arm and hand muscles: Deltoid (DEL), Biceps Brachii (BIC), Triceps Brachii (TRI), Extensor Digitorium Communis (EDC), Flexor Carpi Radialis (FCR), Extensor Carpi Radialis (ECR), Flexor Digitorium Superficialis (FDS). Mk-Br received an additional electrode in the Abductor Pollicis Brevis (ABP). Due to practical constraints, Mk-Sa received electrodes only in Biceps Brachii (BIC), Triceps Brachii (TRI) and Flexor Digitorium Superficialis (FDS). In all animals, wires were then connected to an additional pedestal (Pedestal B), fixated to the titanium mesh.

During the third surgical procedure, monkeys were subjected to a lesion at the cervical level (C5/C6) of the spinal cord. The surgeon used a micro-blade to cut approximately one third of the dorsolateral aspect of the spinal cord, in order to interrupt the main component of the corticospinal tract unilaterally. All monkeys retained autonomic functions, as well as limited arm flexion and shoulder adduction capabilities. We monitored the animals for the first hours after surgery and several times daily during the following days. Monitoring scales were used to assess postoperative pain. Antibiotics were given immediately after the surgery and then once per day for 10 subsequent days, anti-inflammatory drugs were given once per day for 5 days (Rymadyl $4 \mathrm{mg} / \mathrm{kg}$, s.c.; Dexamethasone $0.3 \mathrm{mg} / \mathrm{kg}$, s.c.), and analgesic was given twice per day for 5 days (Temgesic $0.01 \mathrm{mg} / \mathrm{kg}$, i.m.). Within the same procedure, each monkey received a tailored epidural implant. The implant was inserted in the epidural space of the cervical spinal cord, according to methods described in Schiavone $2020^{30}$ and Capogrosso 2018 ${ }^{11}$. The implant was inserted below the T1 vertebra and pulled until it covered spinal segments from C6 to T1. We performed intra-operative electrophysiology in order to assess and refine the implant positioning so that electrodes are aligned to the animal-specific anatomical features. In particular, we verified that single pulses of stimulation delivered from the most rostral and most caudal electrodes elicited contractions in the BIC and FDS muscle respectively. We re-routed the wires subcutaneously in order to connect them to the Pedestal $B$. All surgical and post-operative care procedures are developed in details in previous reports ${ }^{11,40}$. For Mk-Sa, data presented in this paper were collected several weeks pre lesion and 1week post lesion, unfortunately a severe infection of the spinal array and EMGs that recurred after day 7 lead to the premature euthanasia of the monkey before the study could be completed in agreement with the endpoints in our animal authorization. For $\mathrm{Mk}-\mathrm{Br}$ and $\mathrm{Mk}-\mathrm{Yg}$ data presented in this paper were collected several weeks pre lesion and until 3 weeks post lesion.

380 At the end of week 3 post lesion, $\mathrm{Mk}-\mathrm{Br}$ had 2 episodes of self-mutilation on the foot ipsi-lateral to the lesion. In consequence we euthanized the animal before the end of the protocol according to the endpoints in our animal authorization. As described in the results section, we found postmortem that $\mathrm{Mk}-\mathrm{Br}$ had a medial spinal cord contusion at the T3 level. While this lesion did not affect motor control of the legs or the arms, it may have generated neuropathic pain.

\section{Data acquisition}

For Mk-Sa and $\mathrm{Mk}-\mathrm{Br}$, we acquired three-dimensional spatial coordinates of arm and hand joints using a 14-camera motion tracking system (Figure 1, Vicon Motion Systems, Oxford, UK) that tracked the Cartesian position of 6 infrared reflective markers (6 to $9 \mathrm{~mm}$ in diameter each, Vicon Motion Systems, Oxford, UK) at a $100 \mathrm{~Hz}$ framerate. All markers were placed on the left arm, one

390 below the shoulder, three on the elbow (proximal, medial and distal position), and two on the left 
and right side of the wrist. For each subject, a model of the marker placement was calibrated in Vicon's Nexus software at the beginning of each experimental session. For Mk-Yg spatial coordinates of arm and hand joints were recorded using two cameras placed parallel to the sagittal and transversal plane of the animal (Vicon Motion Systems, Oxford, UK). The 3D coordinates of the arm and hand joints were extracted using DeepLabCut ${ }^{41}$. Due to the reduced informative content extracted from the camera parallel to the transverse plane, we then only used 2D coordinates on the animals' sagittal plane. The training set needed for automatic data labeling was created by manually labeling a subset of recorded videos. An investigator was blinded to the experimental condition and was instructed to mark four anatomical landmarks that mirrored the position of markers in Mk-Sa and $\mathrm{Mk}-\mathrm{Br}$ (shoulder, medial elbow, left and right wrist). Neural signals were acquired with a Neural Signal Processor (Blackrock Microsystems, USA) using the Cereplex-E headstage with a sampling frequency of $30 \mathrm{kHz}$. Electromyographic signals were acquired with a Behavioral Neurophysiology chronic recording system (RZ2 BioAmp Processor, Tucker-Davis Technologies, USA) at a sampling frequency of $12207 \mathrm{~Hz}$.

\section{Electrophysiology in sedated monkeys}

Monkeys were sedated with a continuous intravenous infusion of propofol $(5 \mathrm{ml} / \mathrm{kg} / \mathrm{h})$ that minimizes effects on spinal cord stimulation ${ }^{42}$. We delivered single pulses of cathodic, charge balanced, asymmetric square pulses $(0.3 \mathrm{~ms}, 1 \mathrm{~Hz})$ from each electrode contact while recording compound potentials from all implanted arm and hand muscles. Electromyographic signals were acquired with a Behavioral Neurophysiology chronic recording system (RZ2 BioAmp Processor, Tucker-Davis Technologies, USA) at a sampling frequency of $12207 \mathrm{~Hz}$. We then delivered 10 repetitions of pulse trains from each contact, at several frequencies ranging from 20 to $120 \mathrm{~Hz}$. We recorded compound potentials from all implanted arm and hand muscles and arm kinematics through two high resolution cameras (Sony FDR-X3000 Action Cam 4K). Through this procedure we identified three contacts that primarily elicited (1) arm flexors, (2) arm extensors and (3) hand flexors. In a reduced set of trials, we also recorded the force produced by arm flexion through a $10 \mathrm{~N}$ range force sensor (Dual-Range Force Sensor, DFS-BTA, Vernier, Beaverton, Oregon, USA). To record the pulling force produced during isometric arm flexion, the hand was fixated to the sensor hook through a string, and the sensor and the elbow were kept in place by two experimenters, in order to optimally capture the strength produced by muscle contraction.

\section{Behavioral experimental recordings}

All animals were trained to perform a three-dimensional robotic reach, grasp and pull task, previously described in detail in (Barra 201926) and briefly recalled here for simplicity. All animals were instructed to wait for a start signal by resting the left hand on a metallic bar. When the "go-cue" was given, monkeys had to reach for and grasp a small spherical object attached to the robot end effector and located in the three-dimensional space. The object was placed approximately $180 \mathrm{~mm}$ above the animal seating height, $150 \mathrm{~mm}$ far from the shoulder/head coronal plane and $30 \mathrm{~mm}$ left of the animal's left arm. Once animals got a hold on the object, they had to pull it towards their own body until trespassing a virtual spatial threshold. 
The accomplishment of such virtual threshold was automatically detected by the robot control through online monitoring of the end effector position. Once attained the threshold, monkeys had to let go on the object and go back to the metallic bar. Fruits and vegetables were used to reward successful movements. Animals were trained daily (5 days per week) and every session ended as soon as the animals showed any sign of fatigue or impatience.

\section{Stimulation during three-dimensional reach and pull task in injured monkeys}

All monkeys were recorded after injury as soon as they could independently move in their housing, feed themselves autonomously and did not show signs of discomfort. This corresponded to 3, 5 and 6 days after injury respectively for $\mathrm{Mk}-\mathrm{Yg}, \mathrm{Mk}-\mathrm{Br}$ and $\mathrm{Mk}-\mathrm{Sa}$. Each recording session was organized as follows. First, we recorded two blocks without stimulation, each of the duration of approximately 2 minutes. During those blocks we visually evaluated the impairment level of the animal and the performance of the brain decoder. Second, we used the brain decoder to trigger specific stimulation patterns. Contacts used to elicit those functions were defined through the experiments described in the previous paragraph and combined together to create stimulation protocols that allowed the animal to perform a full reach, grasp and pull movement.

\section{Identification and classification of arm movements for kinematic analysis}

We defined the movement performed by the animals as composed of three different phases: reach, grasp and pull. The identification of the reach phase was done by marking the moment in which the left hand left the metallic bar to when the hand closed around the object secured to the robot hand effector (the grasp event). The grasp phase was considered to be a window of 100 $\mathrm{ms}$ around the moment in which hand closed around the object. The pull phase started from the grasp event and finished when the animal accomplished the task by pulling the object across the virtual spatial threshold and placed the hand back on the resting bar. Events related to the 3 phases of the movement (movement onset: reaching, grasp onset: grasping and release of the object, and pulling) were identified manually by inspecting video recordings from Vicon Motion Systems (Oxford, UK). The same method was applied to mark successful and complete performance of reach, grasp and pull movements as events. A successful reach was defined as a complete extension of the arm that brought the hand at the position of the target (even when grasp could not be performed). A successful grasp was defined as a successful closure of the hand around the target. A successful pull was defined as the accomplishment of a complete flexion movement that brought the target past the virtual spatial threshold. Events were then extracted from Vicon and used to perform analysis on the kinematic of the movements and to train the brain decoder by automatic routines (Matlab 2019b). All the analysis was conducted as blinded experiments.

\section{Decoding motor states from intracortical signals}

We designed a neural decoder that detected reaching and grasping events using intracortical 
spiking activity. In order to detect spikes, we set a threshold on each channel of -4 times the rootmean-square voltage recorded during a brief period while the monkey was at rest. We estimated firing rates in each of the motor cortical array channels by summing the multiunit spikes with a $150 \mathrm{~ms}$ history every $0.5 \mathrm{~ms}$. We used these multiunit firing rate estimates to compute a twentydimensional neural manifold capturing the majority of population variance ${ }^{43}$. We projected the spiking activity onto this manifold to calibrate a multiclass regularized linear discriminant analysis decoder ${ }^{40}$ that predicted the labeled timing of reach and grasp events. The decoder used $500 \mathrm{~ms}$ of past neural activity and output the probability of observing the reach and grasp events. During calibration, we defined a probability threshold for each event ranging from 0.8 to 0.99 to optimize predictions of the timing of each event using cross-validation. Since the monkeys could not complete the task after $\mathrm{SCl}$, we were unable to consistently acquire labeled training data. We therefore calibrated a decoding algorithm using reaches from a recording session of a healthy monkey. We then manually labeled attempted reaches after $\mathrm{SCl}$ by manual inspection of video recordings. Using canonical correlation analysis, we aligned the neural dynamics ${ }^{44}$ preceding reaches on the healthy sessions to the observed neural dynamics preceding attempted reaches after SCl. These aligned dynamics were used to control the decoder trained on the healthy reaches.

485 We implemented a custom C++ software application running a control suite that used the 486 decoding algorithm to trigger EES stimulation in real-time. The application received neural data 487 over UDP and made predictions using the decoding algorithm at $15 \mathrm{~ms}$ intervals. When the output 488 probabilities crossed the defined threshold, the application triggered preprogrammed patterns of 489 EES.

\section{$490 \quad$ Analysis of muscle recruitment curves}

491 Electromyographic activity was bandpass filtered between 30 and $800 \mathrm{~Hz}$ with an offline $3^{\text {rd }}$ order Butterworth filter and stimulus artifact were removed. For each animal, stimulation contact, muscle and stimulation amplitude, we extracted compound potentials from 50ms-long segments of electromyographic activity following a stimulation pulse. We then computed the peak-to-peak amplitude of compound potentials. Since we gave four pulses of stimulation for each selected current amplitude, we averaged across values corresponding to the same stimulation amplitude and represented as the mean recruitment value of each muscle as a function of the injected

498 current. For each muscle, recruitment values have been subsequently normalized by the maximum value obtained for that specific muscle, provided that we obtained response saturation

500 (and therefore maximal contraction) in at least one occasion during the session. In addition, we 501 computed a selectivity index for each muscle ${ }^{45}$.

502 In order to obtain a comprehensive measure of muscle recruitment for each contact that would 503 allow to compare across animals, we computed, for each animal, each muscle and each contact, 504 an Average Recruitment Index (ARI) as the average of the recruitment values across all 505 stimulation amplitudes used from a specific stimulation site. 
To compute muscle recruitment during the delivery of pulse train stimulation, we computed the energy of the EMG signal during the duration of stimulation. We then applied the same normalization procedure described above for single pulse recruitment.

Analysis of muscle activity during EES

Electromyographic activity was bandpass filtered between 30 and $800 \mathrm{~Hz}$ with an offline $3^{\text {rd }}$ order Butterworth filter and stimulus artifact were removed. In all animals we computed the energy EMG signals, for each implanted muscle. Energy of EMG signals during stimulation were computed on each segment in which stimulation was delivered after the animal started a movement attempt.

514 Energy of EMG signals without stimulation were computed on each segment in which stimulation was not delivered and the animal started a movement attempt. A movement attempt was defined as an increased EMG activity of the Biceps and Deltoid muscles.

\section{Analysis of kinematics performance}

We performed Principal Component Analysis on a large set of kinematic features. We computed the features on data segments during the reach phase and the pull phase.(see movement identification explained above, section Identification and classification of arm movements for kinematic analysis). All kinematic signals were previously low pass filtered at $6 \mathrm{~Hz}$. Segments were not interpolated nor resampled. Before performing PCA analysis, features were centered to have mean 0 and scaled to have standard deviation of 1 (Matlab 2019). The computed features for $\mathrm{Mk}-\mathrm{Br}$ included: minimum value, maximum value and total excursion of joint angles (shoulder flexion, elbow flexion, and wrist pronation); maximum, minimum and average angular velocity (for the shoulder flexion, elbow flexion and wrist pronation); minimum, maximum and average position along the sagittal, frontal and vertical axis of each arm joint (shoulder, elbow, wrist); maximum minimum and average wrist velocity along the sagittal, frontal and vertical axis; movement smoothness ${ }^{31}$; trajectory length during and time required to complete movements. All the listed features have been computed identically during the reach phase and the pull phase separately and treated as different features. In addition, computed maximal applied three-dimensional pulling force and the average position along the sagittal, frontal and vertical axis of each arm joint (shoulder, elbow, wrist) during grasp;

Since for Mk-Yg we only extracted 2D kinematics on the sagittal plane, the kinematic features for Mk-Yg included: minimum value, maximum value and total excursion of joint angles (shoulder flexion and elbow flexion); maximum and average angular velocity (for the shoulder flexion and elbow flexion); minimum, maximum and average position along the sagittal and vertical axis of each arm joint (shoulder, elbow, wrist); maximum and average wrist velocity along the sagittal and vertical axis; movement smoothness ${ }^{31}$; trajectory length during and time required to complete movements. All the listed features have been computed during the reach phase. 
543 To study how motor cortical activity interacted with EES, we analyzed the neural recordings from $544 \mathrm{Mk}-\mathrm{Br}$ and $\mathrm{Mk}-\mathrm{Yg}$. We identified periods where EES pulse trains produced no discernible 545 movements by setting a threshold on hand velocity. We compared multi-unit neural firing rates on each channel in this period to neural firing rates in the previously identified trials where EES enabled reaching and grasping. First, we counted the number of spikes within the window of stimulation and divided by the duration of stimulation. We then averaged across stimulus repetitions of the movement and no movement conditions and pooled across recording sites in motor cortex.

551 We next computed instantaneous estimates of multi-unit firing rates on each channel by counting 552 the number of spikes in non-overlapping $20 \mathrm{~ms}$ bins and convolving with a gaussian kernel of 50 ms width. We applied Principal Component Analysis (PCA) to compute 10-dimensional neural manifolds spanning this multi-unit population activity ${ }^{43}$. We projected the neural activity onto these manifold axes during the periods where EES evoked either movement or no movement. We then identified periods where the monkey was at rest with no EES, as well as periods where the monkey attempted movements of the arm with no EES. To compare the similarity of neural activity between these conditions, we computed the Mahalananobis distance between activity at rest and the three other periods: EES with movement, EES with no movement, and attempted movements 560 with no EES.

\section{Histology}

562 Monkeys were deeply anesthetized (lethal dose of pentobarbital, $60 \mathrm{mg} / \mathrm{kg}$, injected i.v.) and transcardially perfused with saline (about $200 \mathrm{ml}$ ), followed by 3 liters of $4 \%$ paraformaldehyde (PFA). Dissected spinal cord were post-fixed in 4\% PFA overnight, and then immersed in $30 \%$ sucrose solution for 2 weeks. $50 \mu \mathrm{m}$ transverse or horizontal sections were cut using a cryostat and kept in $0.1 \mathrm{M}$ PBS azide $(0.03 \%)$ at $4^{\circ} \mathrm{C}$. Primary antibodies were: rabbit anti-lba1 (1:1000, Wako) and guinea pig anti-NeuN (1:300, Millipore). Fluorescence secondary antibodies were conjugated to: Alexa fluor 647 and Alexa fluor 555 (Life technologies). Sections were coverslipped using Mowiol. Immunofluorescence was imaged digitally using a slide scanner (Olympus VS-120). Lesions were reconstructed using image analysis software (Neurolucida) to trace the lesion over serial sections (200 $\mu \mathrm{m}$ apart).

\section{Statistical procedures:}

All data are reported as mean values \pm standard error of the mean (s.e.m.) or mean values \pm standard deviation (std). The choice is highlighted directly in the figures or in the relative caption. Significance was analyzed using the non-parametric Wilcoxon rank-sum test. In only one case ${ }^{*} \mathrm{p}<0.05,{ }^{* *} \mathrm{p}<0.01,{ }^{* * *} \mathrm{p}<0.001$.

\section{References}


1. ICCP. International Campaign for Cures of Spinal Cord Injury Paralysis. http://www.campaignforcure.org.

2. Anderson, K. D. Targeting recovery: priorities of the spinal cord-injured population. Journal of neurotrauma 21, 1371-1383 (2004).

3. Lemon, R. N. Descending pathways in motor control. Annual review of neuroscience 31, $585 \quad$ 195-218 (2008).

4. Lebedev, M. A. \& Nicolelis, M. A. Brain-machine interfaces: From basic science to neuroprostheses and neurorehabilitation. Physiological reviews (2017).

5. Moritz, C. T., Perlmutter, S. I. \& Fetz, E. E. Direct control of paralysed muscles by cortical neurons. Nature 456, 639-642 (2008).

6. Ethier, C., Oby, E. R., Bauman, M. J. \& Miller, L. E. Restoration of grasp following paralysis through brain-controlled stimulation of muscles. Nature 485, 368-371 (2012).

7. Bouton, C. E. et al. Restoring cortical control of functional movement in a human with quadriplegia. Nature (2016) doi:10.1038/nature17435.

8. Ajiboye, A. B. et al. Restoration of reaching and grasping movements through braincontrolled muscle stimulation in a person with tetraplegia: a proof-of-concept demonstration. The Lancet (2017) doi:10.1016/S0140-6736(17)30601-3.

9. Giat, Y., Mizrahi, J. \& Levy, M. A musculotendon model of the fatigue profiles of paralyzed quadriceps muscle under FES. IEEE transactions on biomedical engineering 40, 664-674 (1993).

600 research 24, 443-452 (2002). 
12. Angeli, C. A. et al. Recovery of Over-Ground Walking after Chronic Motor Complete Spinal

$605 \quad$ Cord Injury. N Engl J Med 379, 1244-1250 (2018).

13. Gill, M. L. et al. Neuromodulation of lumbosacral spinal networks enables independent stepping after complete paraplegia. Nat Med (2018) doi:10.1038/s41591-018-0175-7.

14. Wagner, F. B. et al. Targeted neurotechnology restores walking in humans with spinal cord $609 \quad$ injury. Nature 563, 65 (2018).

610 15. Holinski, B. J. et al. Intraspinal microstimulation produces over-ground walking in

611 anesthetized cats. J Neural Eng 13, 056016 (2016).

612 16. Zimmermann, J. B., Seki, K. \& Jackson, A. Reanimating the arm and hand with intraspinal 613 microstimulation. Journal of neural engineering 8, 054001 (2011).

614 17. Gaunt, R. A., Prochazka, A., Mushahwar, V. K., Guevremont, L. \& Ellaway, P. H. Intraspinal microstimulation excites multisegmental sensory afferents at lower stimulus levels than local a-motoneuron responses. Journal of neurophysiology 96, 2995-3005 (2006).

18. Wenger, N. et al. Closed-loop neuromodulation of spinal sensorimotor circuits controls refined locomotion after complete spinal cord injury. Science translational medicine $\mathbf{6}$,

19. Harkema, S. et al. Effect of epidural stimulation of the lumbosacral spinal cord on voluntary movement, standing, and assisted stepping after motor complete paraplegia: a case study. The Lancet 377, 1938-1947 (2011).

20. Angeli, C. A., Edgerton, V. R., Gerasimenko, Y. P. \& Harkema, S. J. Altering spinal cord excitability enables voluntary movements after chronic complete paralysis in humans. Brain 137, 1394-1409 (2014).

21. Lu, D. C. et al. Engaging Cervical Spinal Cord Networks to Reenable Volitional Control of Hand Function in Tetraplegic Patients. Neurorehabil Neural Repair 30, 951-962 (2016). 
628 22. Nishimura, Y., Perlmutter, S. I. \& Fetz, E. E. Restoration of upper limb movement via

629 artificial corticospinal and musculospinal connections in a monkey with spinal cord injury.

$630 \quad$ Frontiers in neural circuits 7, 57 (2013).

631 23. Weiler, J., Gribble, P. L. \& Pruszynski, J. A. Spinal stretch reflexes support efficient hand 632 control. Nat. Neurosci 1-11 (2019).

633 24. Kinoshita, M. et al. Genetic dissection of the circuit for hand dexterity in primates. Nature 487, 235-238 (2012).

25. Alstermark, B. \& Isa, T. Circuits for skilled reaching and grasping. Annu Rev Neurosci 35, 559-78 (2012).

637 26. Barra, B. et al. A Versatile Robotic Platform for the Design of Natural, Three-Dimensional

638 Reaching and Grasping Tasks in Monkeys. Journal of Neural Engineering (2019) doi:10.1088/1741-2552/ab4c77.

27. Jenny, A. B. \& Inukai, J. Principles of motor organization of the monkey cervical spinal cord. J Neurosci 3, 567-75 (1983).

642 28. Greiner, N. et al. Recruitment of Upper-Limb Motoneurons with Epidural Electrical Stimulation of the Primate Cervical Spinal Cord. bioRxiv (2020).

644 29. Capogrosso, M. et al. A computational model for epidural electrical stimulation of spinal sensorimotor circuits. The Journal of neuroscience : the official journal of the Society for

647 30. Schiavone, G. et al. Soft, Implantable Bioelectronic Interfaces for Translational Research. Advanced Materials 32, 1906512 (2020).

649 31. Teulings, H.-L., Contreras-Vidal, J. L., Stelmach, G. E. \& Adler, C. H. Parkinsonism 650 Reduces Coordination of Fingers, Wrist, and Arm in Fine Motor Control. Experimental $651 \quad$ Neurology 146, 159-170 (1997). 
652 32. Sharpe, A. N. \& Jackson, A. Upper-limb muscle responses to epidural, subdural and 653 intraspinal stimulation of the cervical spinal cord. J Neural Eng 11, 016005 (2014).

654 33. Hofstoetter, U. S. et al. Periodic modulation of repetitively elicited monosynaptic reflexes of 655 the human lumbosacral spinal cord. Journal of neurophysiology jn 001362015 (2015) 656 doi:10.1152/jn.00136.2015.

657 34. Moritz, C. T., Lucas, T. H., Perlmutter, S. I. \& Fetz, E. E. Forelimb movements and muscle 658 659

35. Moraud, E. M. et al. Mechanisms Underlying the Neuromodulation of Spinal Circuits for 661 Correcting Gait and Balance Deficits after Spinal Cord Injury. Neuron 89, 814-28 (2016). 662 36. Ting, J. et al. A wearable neural interface for detecting and decoding attempted hand 663 movements in a person with tetraplegia. in 1930-1933 (IEEE, 2019).

664 37. Griffin, D. M., Hoffman, D. S. \& Strick, P. L. Corticomotoneuronal cells are 'functionally 665 tuned'. Science 350, 667-70 (2015).

666 38. Griffin, D. M. \& Strick, P. L. The motor cortex uses active suppression to sculpt movement. 667 Sci Adv 6, eabb8395 (2020).

668 39. National Research Council (US) Institute for Laboratory Animal Research. Guide for the 669 Care and Use of Laboratory Animals. (National Academies Press (US), 1996).

670 40. Capogrosso, M. et al. A brain-spine interface alleviating gait deficits after spinal cord injury 671 in primates. Nature 539, 284-288 (2016).

672 41. Mathis, A. et al. DeepLabCut: markerless pose estimation of user-defined body parts with 673 deep learning. Nature neuroscience 21, 1281-1289 (2018).

674 42. Toossi, A. et al. Effect of anesthesia on motor responses evoked by spinal neural 675 prostheses during intraoperative procedures. Journal of neural engineering 16, 036003 676 (2019). 
677 43. Gallego, J. A., Perich, M. G., Miller, L. E. \& Solla, S. A. Neural Manifolds for the Control of $678 \quad$ Movement. Neuron 94, 978-984 (2017).

679 44. Gallego, J. A., Perich, M. G., Chowdhury, R. H., Solla, S. A. \& Miller, L. E. Long-term 680 stability of cortical population dynamics underlying consistent behavior. Nature 681 neuroscience 23, 260-270 (2020).

682 45. Raspopovic, S., Capogrosso, M. \& Micera, S. A Computational Model for the Stimulation of 683 Rat Sciatic Nerve Using a Transverse Intrafascicular Multichannel Electrode. IEEE 684 Transactions on Neural Systems and Rehabilitation Engineering 19, 333-344 (2011). 


\section{Figures}
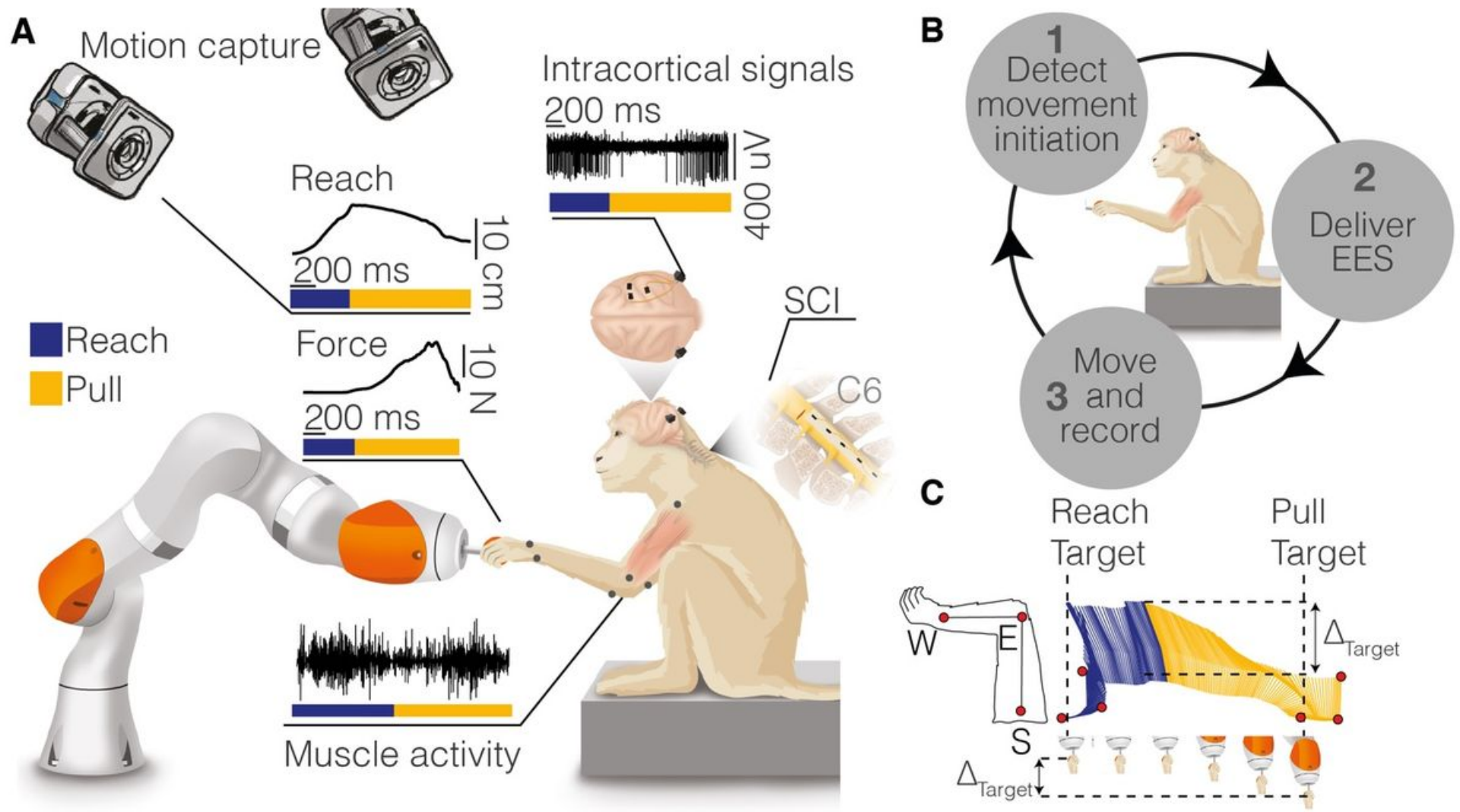

Figure 1

Experimental framework. (A) Monkeys were trained to reach for, grasp, and pull a target object placed at the end effector of a robotic arm. We measured 3D forces applied to the robot joints, full-limb kinematics, electromyographic (EMG) activity from eight muscles of the arm and hand, and intracortical signals from primary sensorimotor areas. (B) Conceptual scheme of the experimental protocol: (1) A decoder running on a control computer identified movement attempts and (2) delivered electrical spinal cord stimulation to the appropriate spinal roots. (3) Stimulation produced arm and hand movement that we recorded and analyzed off-line. (C) Stick diagram decomposition of arm movement during a reach, grasp and pull movement in intact monkeys ( $\mathrm{S}=$ shoulder, $\mathrm{E}$ = elbow, $\mathrm{W}=$ wrist). We considered a movement complete when a target spatial threshold was crossed during pull. Copyright Jemère Ruby. 
A

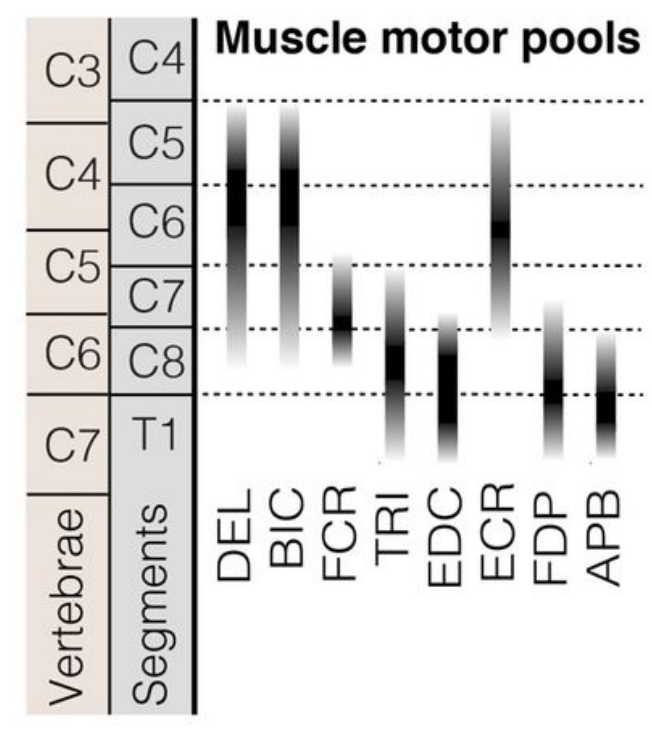

B

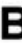

B

Electrode implantation

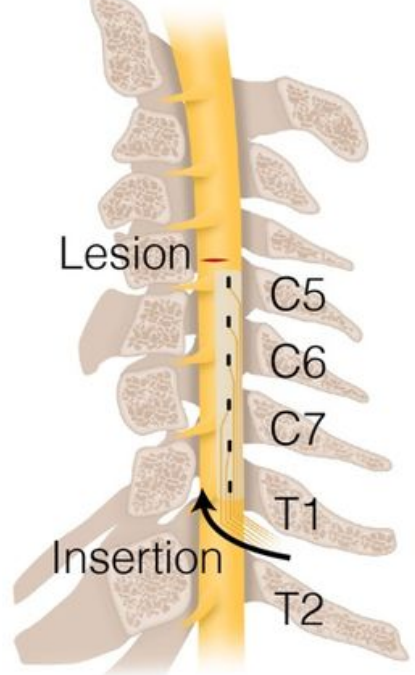

D
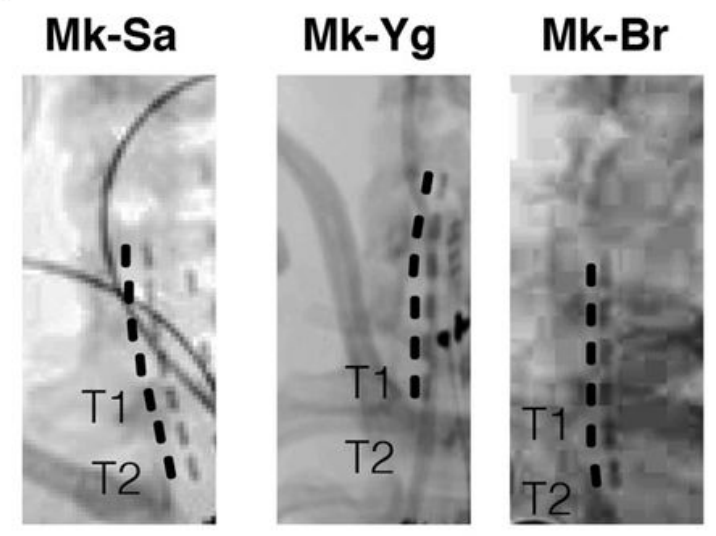

Anatomical landmarks
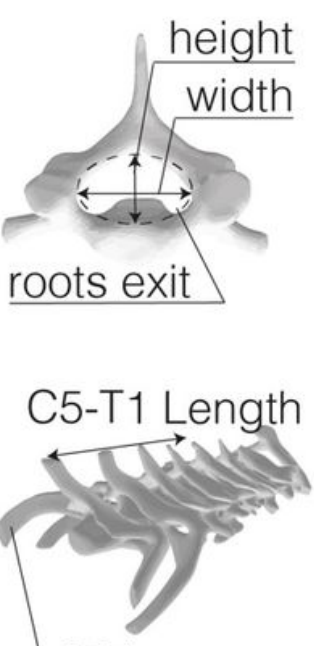

CT bone reconstruction

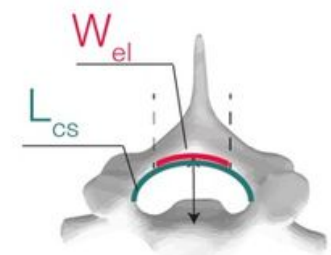

Personalization landmarks

$$
W_{e l}=1 / 3 L_{c s}
$$

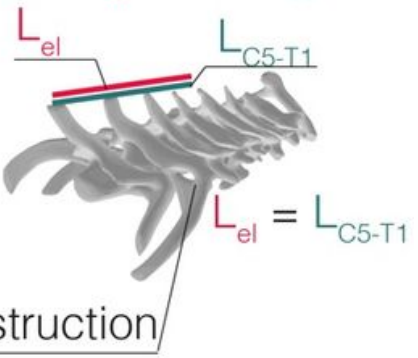

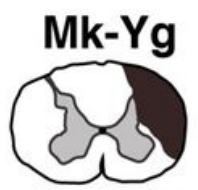

Lesion 19,5\%

Grey M.

$\square$ White M.

Lesion

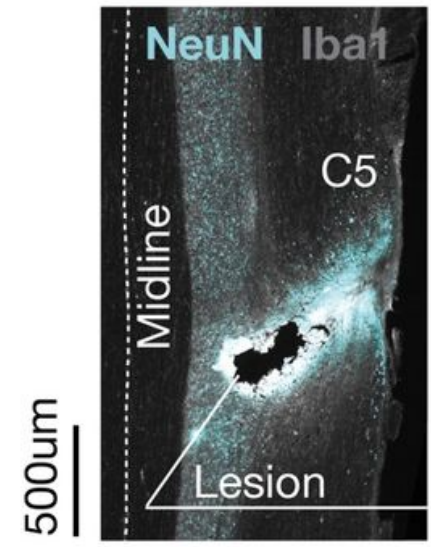

Figure 2

Epidural electrode design and implantation. (A) Motoneurons pool distribution of arm and hand muscles in the cervical spinal cord in relation to vertebrae and spinal segments (adapted from Jenny and Inukai, 1983). Deltoid (DEL), Biceps Brachii (BIC), Flexor Carpi Radialis (FCR), Triceps Brachii (TRI), Extensor Digitorium Communis (EDC), Extensor Carpi Radialis (ECR), Flexor Digitorium Profundis (FDP), Abductor Pollicis Brevis (ABP). (B) Schematic representation illustrating the positioning and insertion of the spinal implant in the epidural space; on the right, anatomical landmarks used to tailor the epidural interface to each monkey's anatomy (Length of dorsal aspect of spinal canal Lcs, length of C5-T1 spinal segment LC5-T1, electrode width Wel, electrode length Lel). Three-dimensional reconstructions of vertebras are obtained by CT-reconstruction (Osirix, Pixmeo, Switzerland). (C) Representative X-ray scans of the epidural implant in the three monkeys (Mk-Sa, Mk-Br and Mk-Yg). (D) Anatomical reconstruction of the cervical spinal cord lesion (black area) for the 3 monkeys, shown on a transversal section. On the right, representative image of longitudinal section of the spinal cord of $\mathrm{Mk}-\mathrm{Br}$ around the lesion site stained with NeuN (neuronal cell bodies) and Iba1 (microglia). 
A Muscle selectivity during single pulse

Selectivity index : $0 \square 0.650 \square \square .7$

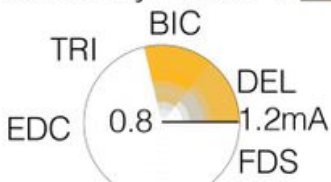
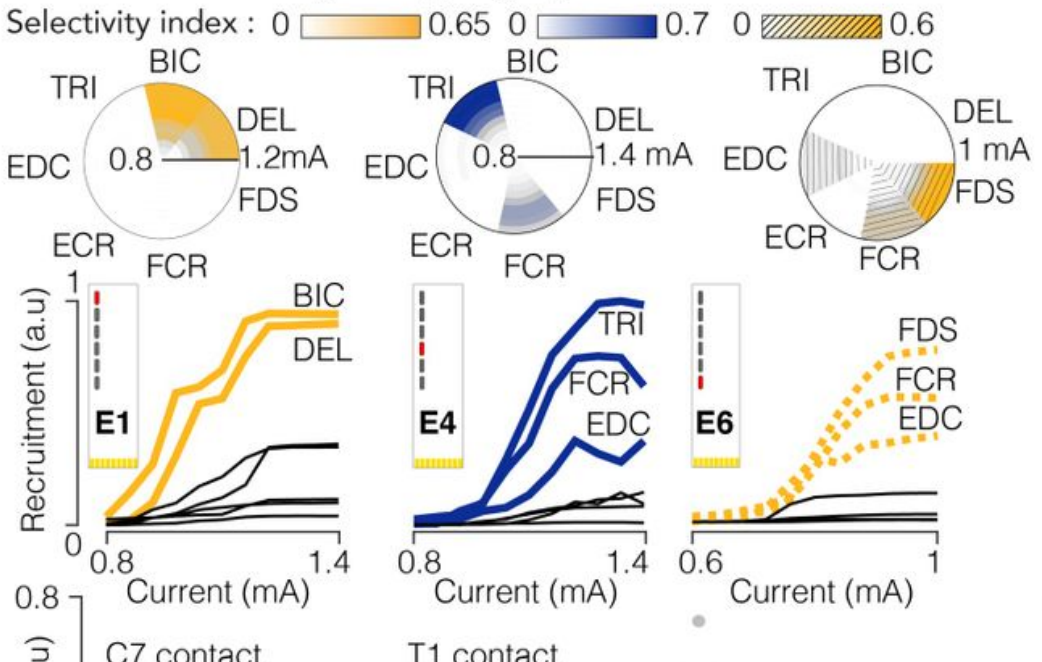

Current (mA)

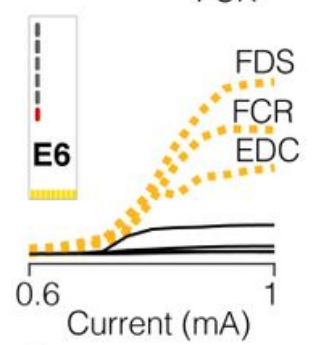

T1 contact

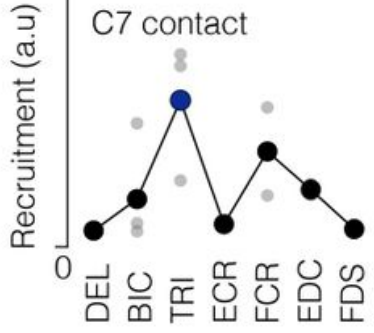

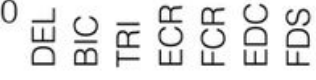

C Functional kinematics

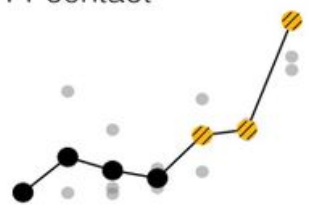

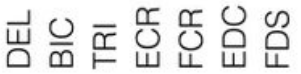

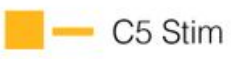

- C6/C7 Stim. VIA... T1 Stim.
B Muscle selectivity during pulse trains
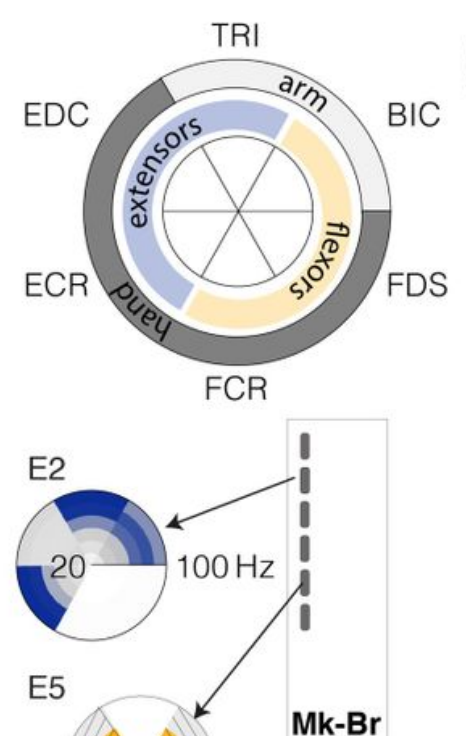

$\mathrm{Mk}-\mathrm{Br}$

ичичиน

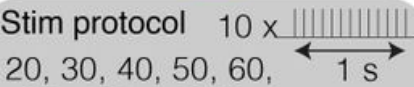
$80,100,120 \mathrm{~Hz}$

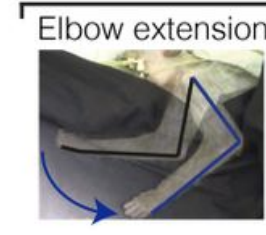

Wrist flexion

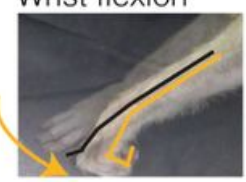

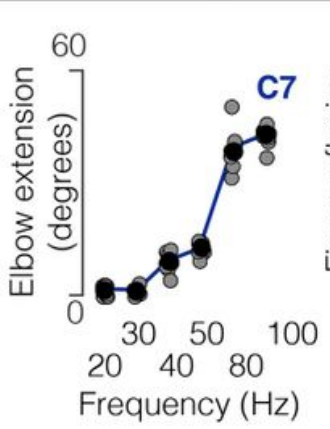

Frequency $(\mathrm{Hz})$

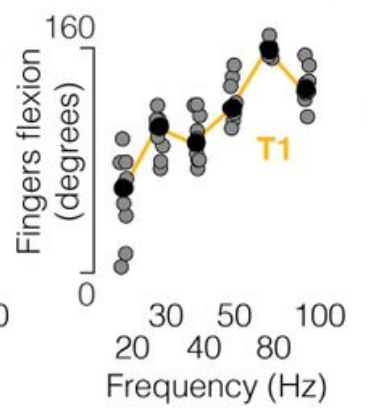

Frequency $(\mathrm{Hz})$

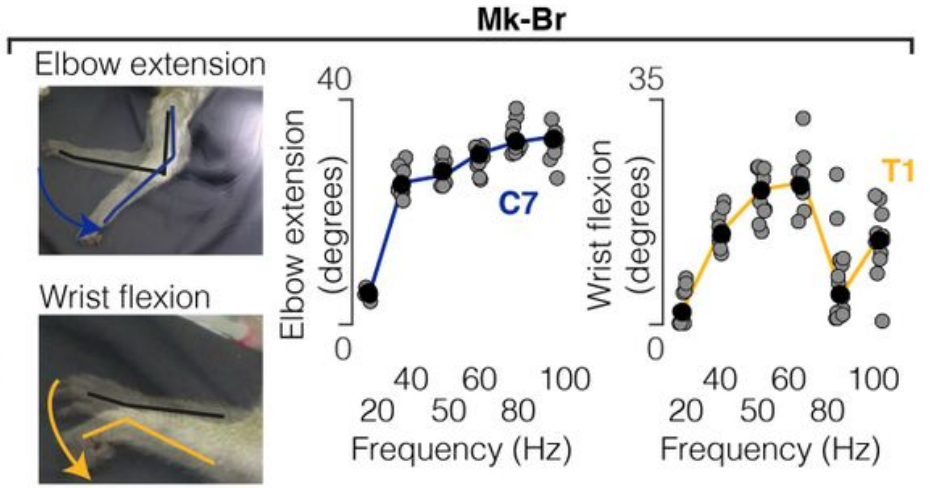

Figure 3

EES produces single joint movements in anesthetized animals. (A) Examples of muscle selectivity (polar plot) and muscle recruitment obtained by stimulating $(1 \mathrm{~Hz})$ at $\mathrm{C} 5, \mathrm{C} 6 / \mathrm{C} 7$, and $\mathrm{T} 1$ spinal segments (Mk$\mathrm{Yg}$ ). Below, average muscle activations elicited from $\mathrm{C} 7$ and $\mathrm{T} 1$ contacts in $\mathrm{n}=3$ monkeys (Grey bullets: for each animal, average recruitment across all stimulation currents. Big bullets: mean of average recruitments across animals). (B) Muscle recruitment obtained during delivery of pulse trains in anesthetized monkeys. Recruitment was estimated by computing the energy of EMG signals for each muscle and each stimulation contact. Stimulation frequencies ranged from 20 to $120 \mathrm{~Hz}(\mathrm{n}=2)$. For each muscle, energy values were normalized to the maximum value obtained across all frequencies and contacts. (C) Single joint angles excursions induced by stimulation at C7 (blue) and T1 (yellow) of the mean values. 


\section{A Frequency selection}

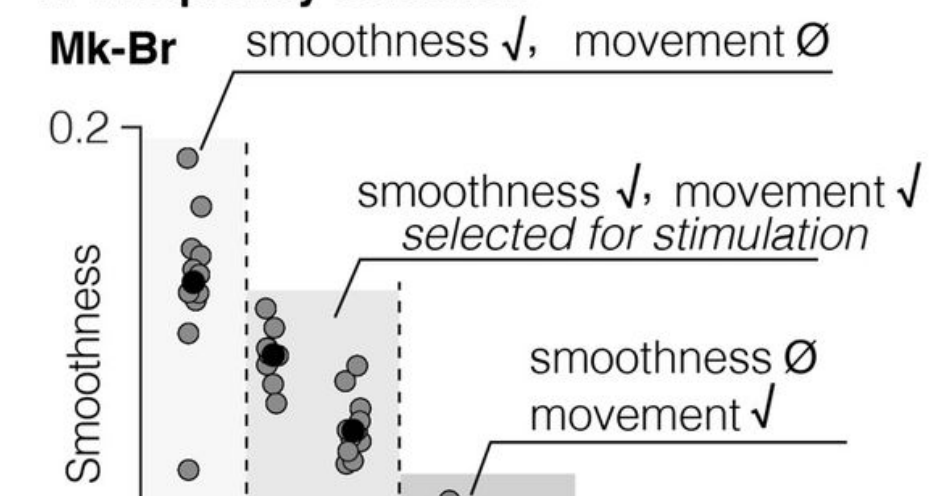

B Electrode selection

Reach Grasp

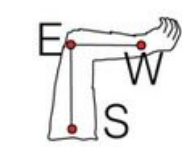

elbow extension

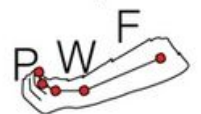

hand and wrist flexion
Pull

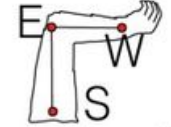

elbow

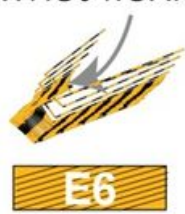
flexion
C INTACT - NO STIM

○
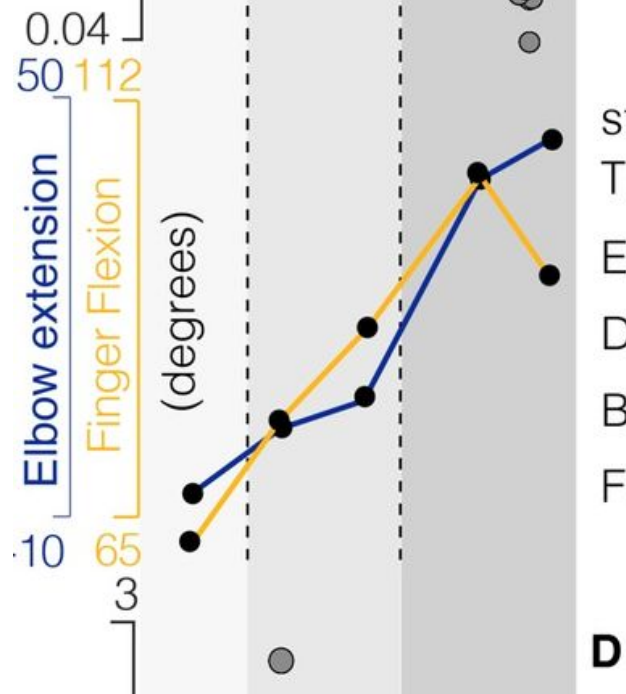

$20 \quad 40 \quad 50 \quad 80 \quad 100$ Frequency $(\mathrm{Hz})$

\section{Stimulation protocol}

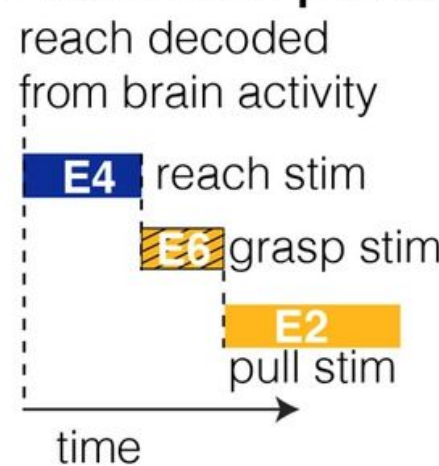

Mk-Br

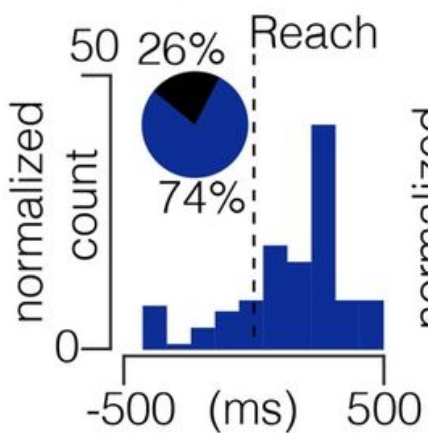

ANESTHETIZED - STIM extension grasp flexion

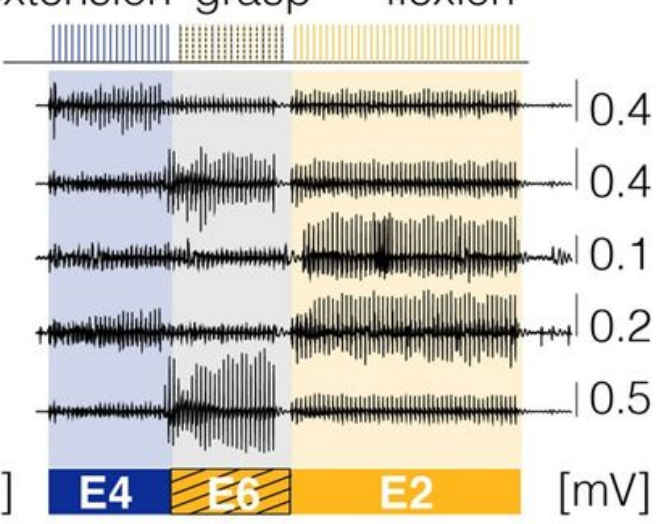

Mk-Yg

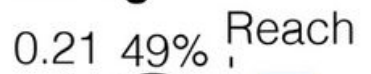

$0.2149 \%$ Reach

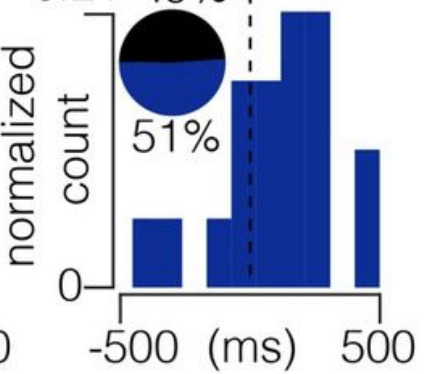

Figure 4

Design of stimulation protocol. (A) Combined representation of movement smoothness, elbow and finger flexion, and pulling force during anesthetized stimulation. Shades of gray highlight three frequency ranges that produce: (1) smooth trajectory, but little movement and low force $(2 \mathrm{~Hz}),(2)$ smooth trajectory, extended movement and medium force (40 and $50 \mathrm{~Hz}),(3)$ abrupt and very extended movement and low force $(80$ and $100 \mathrm{~Hz}$ ). The range $40-50 \mathrm{~Hz}$ was selected as the best optimization of sufficient movement, smoothness and force production. (B) Schematic representation of arm and hand kinematics during stimulation delivered from the selection of three contacts to produce elbow extension (blue), hand and wrist flexion (yellow and black), and elbow flexion (yellow). (C) Example of comparison between EMG activity during intact movement (left) and movement elicited by chaining stimulation from the three 
selected contacts (right). (D) Scheme illustrating how stimulation is triggered from movement-related intra-cortical signals. On the right, online performances of movement attempt decoder in two animals with SCl. Pie charts represent percentage of predicted (blue) and unpredicted (black) reach events by our decoder.

A

Intact

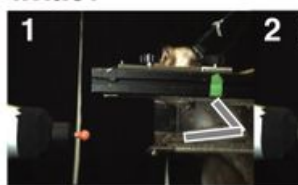

After SCl - stim OFF

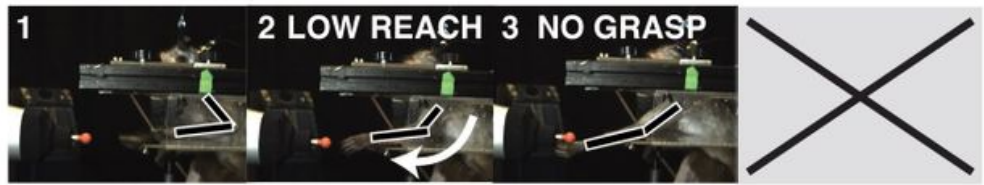

After $\mathrm{SCl}$ - stim ON

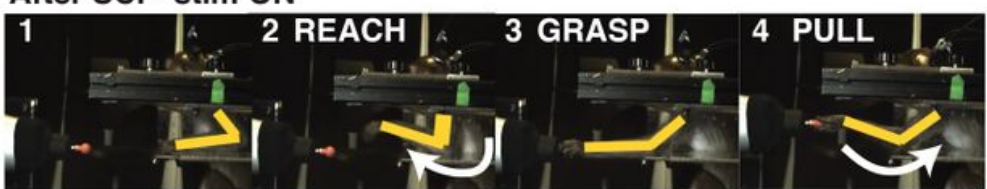

C EES improves task performance Mk-Br, week 3

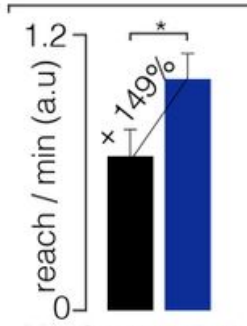

Mk-Sa, week 1
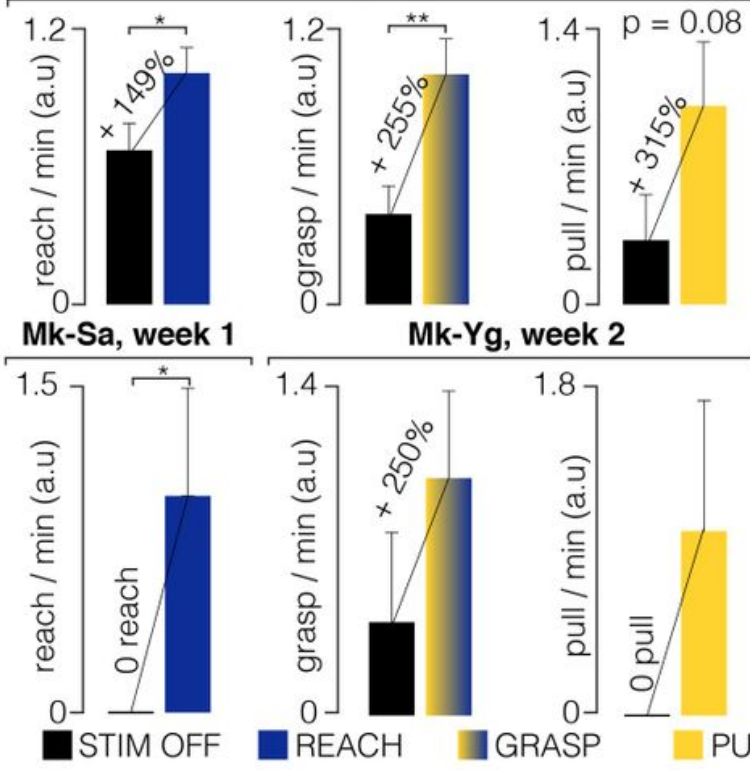

Mk-Yg, week 2

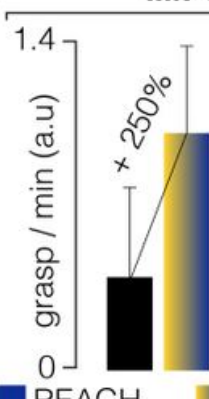

$\mathrm{REACH}$
STIM O

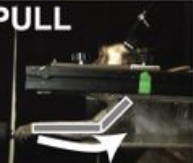

西

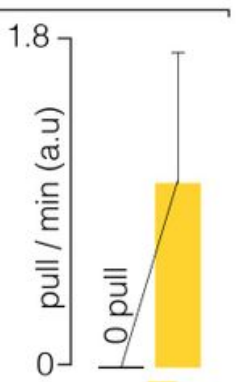

D EES improves kinematics

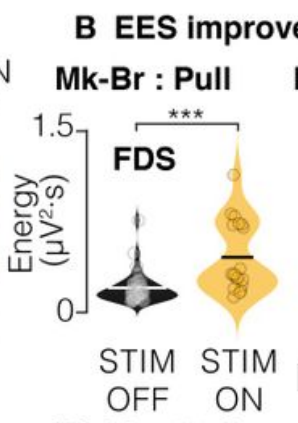

Mk-Yg : Pull

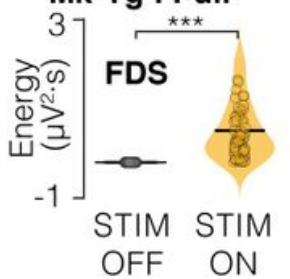

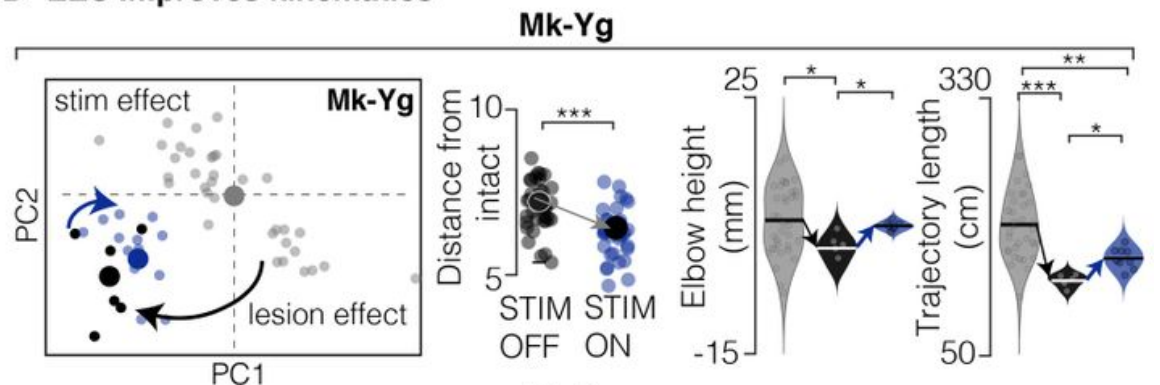
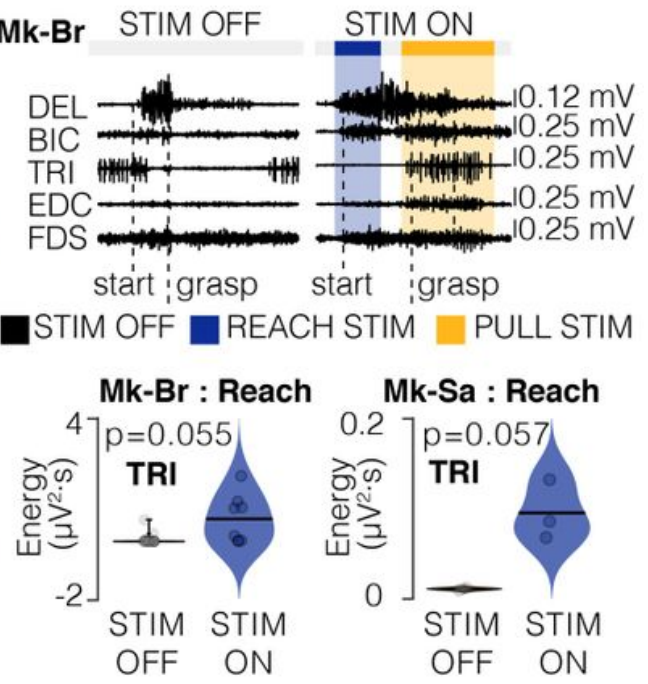

Mk-Yg
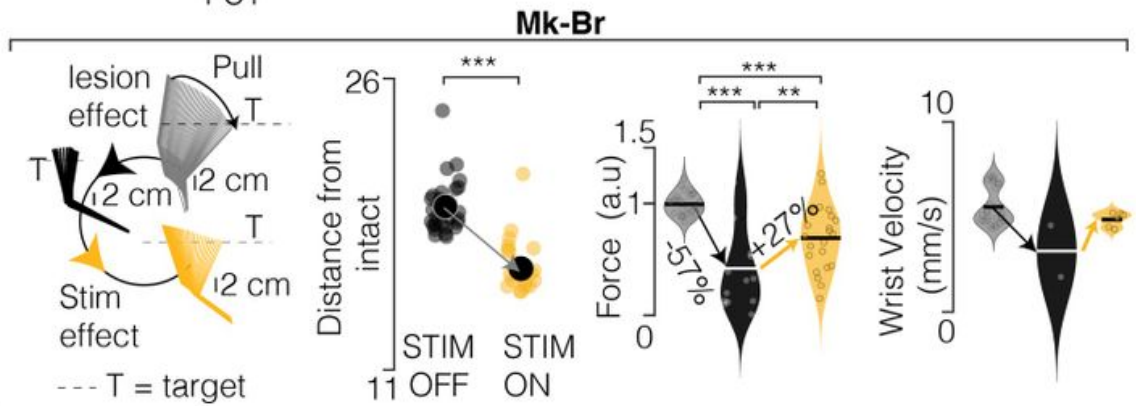

\section{Figure 5}

EES improves task performance, muscle strength and movement quality. (A) Snapshots of Mk-Yg performing the task before $\mathrm{SCl}$, after $\mathrm{SCl}$ without $\mathrm{EES}$, and after $\mathrm{SCl}$ with $\mathrm{EES}$. A full successful trial is composed of a reach, a grasp, and a pull. After $\mathrm{SCl}, \mathrm{Mk}-Y \mathrm{~g}$ could only perform reaching movements without EES, while when EES was delivered the full task could be performed. (B) Violin plots of signal energy of triceps and FDS EMG profiles during reach ( $\mathrm{Mk}-\mathrm{Br}$ and $\mathrm{Mk}-\mathrm{Sa}$ ) and pull ( $\mathrm{Mk}-\mathrm{Br}$ and $\mathrm{Mk}$ $\mathrm{Yg}$ ). All individual data points are represented by bullets. Black lines correspond to means of the distribution. Statistical analysis with Wilcoxon Ranksum test. On the right, example raw EMG data after $\mathrm{SCl}$ with and without EES. (C) Bar plots report the rate of successful movements after SCl, without and 
with stimulation. Data are presented as mean \pm STD and normalized on the mean value in stimulation condition. Statistics was performed with Bootstrap. (D) Example PC analysis of kinematic features (See methods). Top-left, first and second PC space. Bottom left, stick diagram representation of arm kinematics during pull in intact conditions, after SCl without and with EES. At the immediate right (both bottom and top), euclidean distance in the feature space of trials without stimulation (black) and with stimulation (blue) from the centroid of the trials in intact condition. At the extreme right, example violin plots of movement quality features in the three conditions: intact, after $\mathrm{SCl}$, and after $\mathrm{SCl}$ with stimulation. Statistics with Wilcoxon Ranksum test. Asterisks: ${ }^{*} \mathrm{p}<0.05,{ }^{* *} \mathrm{p}<0.01,{ }^{* * *} \mathrm{p}<0.001$.

\section{A Stimulation is gated by sensory feedback}

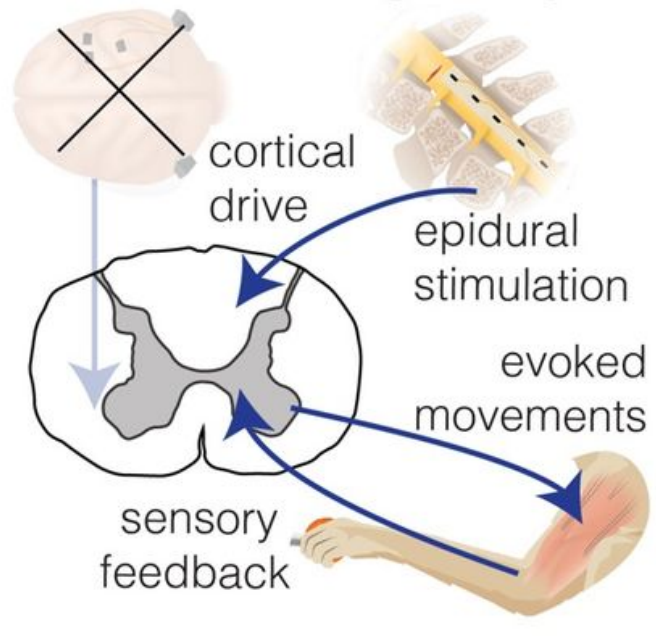

- o Noload O Load
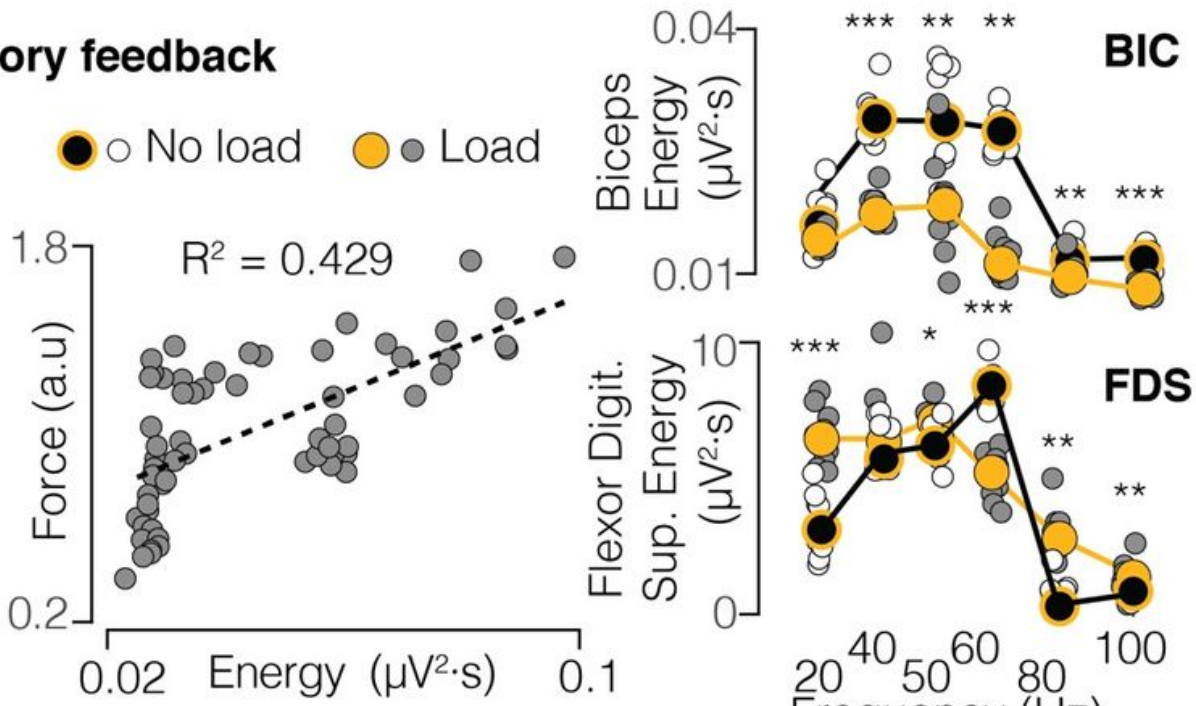

B

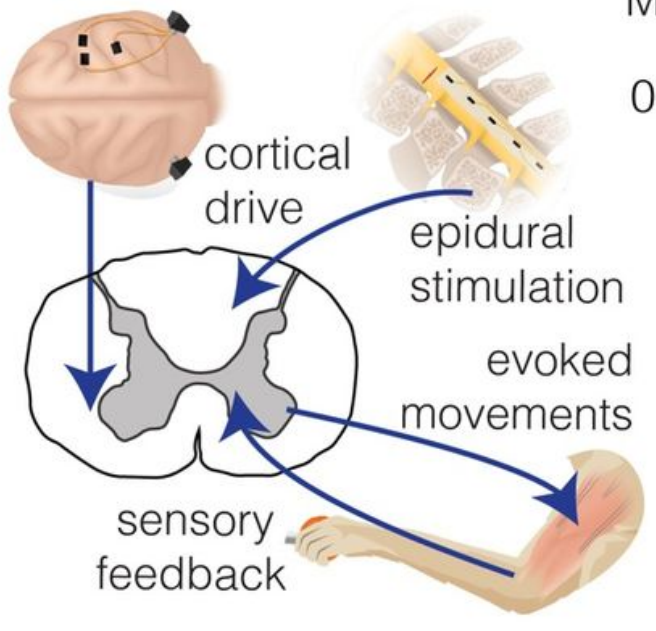

\section{Stimulation is gated by cortical drive}

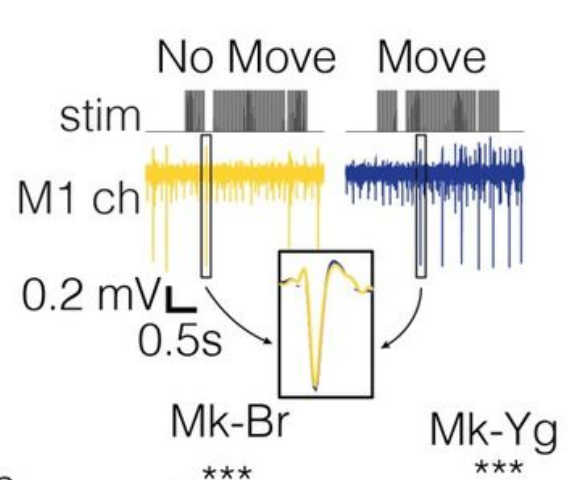

C
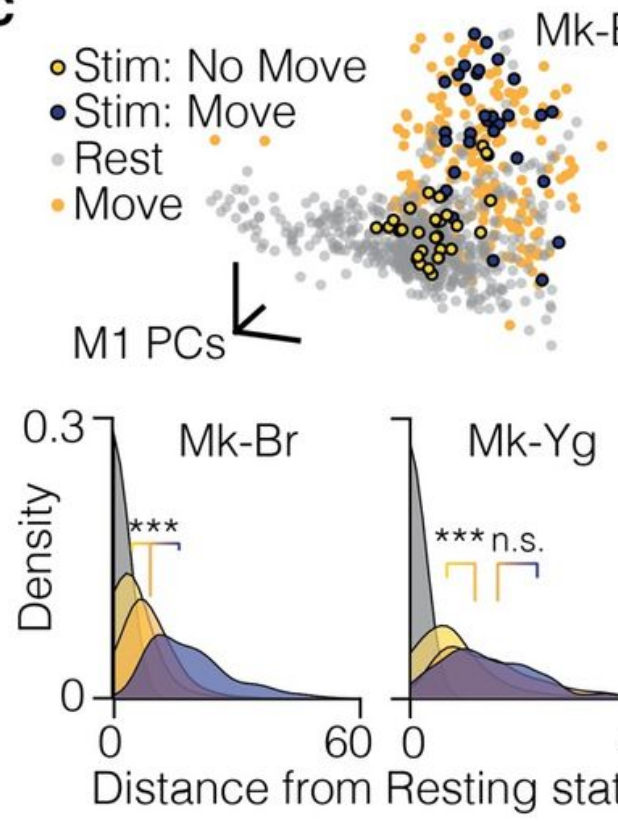

Frequency $(\mathrm{Hz})$ 
energy of the EMG trace of the biceps muscle and arm flexion force produced by muscle contraction during a isometric arm flexion induced by stimulation. Right: energy of EMG signal of biceps and FDS muscles during free arm flexion (no load) or isometric arm flexion (load) induced by stimulation. White and grey bullets: individual data points for no load and load conditions. Black and yellow bullets: mean values for no load and load conditions. Black and yellow lines: interpolation of mean values for no load and load conditions. (B) (Top) The same EES pulse train (top) applied to $\mathrm{Mk}$ - $\mathrm{Br}$ can result in different motor output. For an example M1 channel, the stimulation that evoked movement (blue, right) corresponded to more spiking activity than the same stimulation evoking no movement (yellow, left). (Bottom) Distribution of average firing rates across all M1 channels during stimulation trains that evoked no movement (yellow) and movement (blue). (C) (Top) State space view of M1 activity for all time points during rest (gray) and preceding attempted movement (orange). The brain states during successful stimulation (blue) were similar to those preceding attempted movements, while the unsuccessful stimulation (yellow) overlapped with the rest states. (Bottom) We computed a relative Mahalanobis distance between the two stimulation conditions and the cluster of neural states at rest. For both monkeys, neural states during stimulation periods with no movement were close to rest.

\section{Supplementary Files}

This is a list of supplementary files associated with this preprint. Click to download.

- Video1.mp4

- SupplementaryData.pdf

- Video2.mp4

- Video3.mp4

- Video4.mp4 